\title{
Position, Energy, and Transit Time Distributions in a Hemispherical Deflector Analyzer with Position Sensitive Detector
}

\author{
Omer Sise $^{1}$ and Theo J. M. Zouros ${ }^{2,3}$ \\ ${ }^{1}$ Department of Science Education, Faculty of Education, Suleyman Demirel University, 32260 Isparta, Turkey \\ ${ }^{2}$ Department of Physics, University of Crete, P.O. Box 2208, 71003 Heraklion, Greece \\ ${ }^{3}$ Tandem Lab, INPP, NCSR Demokritos, P.O. Box 60228, 15310 Agia Paraskevi, Greece \\ Correspondence should be addressed to Omer Sise; omersise@gmail.com
}

Received 28 January 2015; Revised 11 May 2015; Accepted 12 May 2015

Academic Editor: Paola Luches

Copyright (C) 2015 O. Sise and T. J. M. Zouros. This is an open access article distributed under the Creative Commons Attribution License, which permits unrestricted use, distribution, and reproduction in any medium, provided the original work is properly cited.

\begin{abstract}
Practical analytic equations, for the ideal field, and numerical results from SIMION simulations, for the fringing field, are presented for the exit radius $r_{\pi}$ and transit time $t_{\pi}$ of electrons in a hemispherical deflector analyzer (HDA) over a wide range of analyzer parameters. Results are presented for a typically dimensioned HDA with mean radius $\bar{R}=101.6 \mathrm{~mm}$ and interradial separation $\Delta R=R_{2}-R_{1}=58.4 \mathrm{~mm}$ able to accommodate a $40 \mathrm{~mm}$ diameter position sensitive detector (PSD). Results for three different entry positions $R_{0}$ are compared: $R_{0}=\bar{R}$ (the conventional central entry) and two displaced (paracentric) entries: $R_{0}=82.55 \mathrm{~mm}$ and $R_{0}=116 \mathrm{~mm}$. Exit spreads $\Delta r_{\pi}, \Delta t_{\pi}$ and base energy resolution $\Delta E_{B}$ are computed for HDA pass energies $E_{0}=10,100,500$, and $1000 \mathrm{eV}$, entry aperture sizes $\Delta r_{0} \leq 1.5 \mathrm{~mm}$, entry angular spreads $\left|\alpha_{\max }\right| \leq 5^{\circ}$, and an electron beam with relative energy spread $\delta E / E_{0} \leq 0.4 \%$. Overall, under realistic conditions, both paracentric entries demonstrate near ideal field behavior and clear superiority over the conventional entry at $R_{0}=\bar{R}$. The $R_{0}=82.55 \mathrm{~mm}$ entry has better absolute energy and time spread resolutions, while the $R_{0}=116 \mathrm{~mm}$ has better relative energy resolutions, both offering attractive alternatives for time-of-flight and coincidence applications where both energy and timing resolutions are important.
\end{abstract}

\section{Introduction}

The hemispherical deflector analyzer (HDA) has become increasingly popular in electron spectroscopy (see [1-6] and references therein), due to several advantages including (a) superior energy resolution approaching the sub-meV level [6], (b) the use of flexible transfer lens systems $[3,6]$ that can be operated in different modes, optimizing selectively transmission, spatial resolution, or angular resolution, and (c) high efficiency by using a fast, high-resolution two-dimensional position sensitive detector (PSD). The simultaneous recording of hundreds of energy channels along the dispersive axis enables the acquisition of high-resolution electron spectra across a large (typically $10-15 \%$ of the tuning energy) kinetic energy window without the need to voltage sweep the analyzer, thus cutting down tremendously on overall data acquisition times (see $[6,7]$ and references therein). In addition, its high time-resolution capabilities enable measurements at the time-of-flight (TOF) spread limit of the HDA [8].

For conventional electron spectroscopy, the flight time of electrons in an analyzer of this type is unimportant. However, if an HDA is used to record phenomena varying rapidly in time as in synchrotron and free-electron laser (FEL) investigations [8], or if it is coupled to another detector in an electron-electron coincidence setup $[9,10]$, the travel times of the electrons through the analyzer become an important point of consideration. The use of an HDA in this type of electronelectron coincidence experiments is possible under certain restrictions: Due to the time-of-flight spread in the HDA, a compromise between energy resolution and time resolution must be made, which renders the higher energy resolutions at low pass energies unusable. For coincidence experiments, pass energies of $\sim 100 \mathrm{eV}$ are typical, having energy resolutions of $\sim 100 \mathrm{meV}$ and time-of-flight spreads of $\sim 4 \mathrm{~ns}$ [10]. 
Recent interest in the use of the HDA has been generated primarily from fields such as angle-resolved photoemission spectroscopy (ARPES) and time-resolved-X-ray photoelectron spectroscopy (tr-XPS). In addition, technological advances in photon sources which can deliver bright, coherent, polarized, monochromatic, or ultrashort stroboscopic light including intense femtosecond lasers, 4th generation synchrotrons [11], and ultramodern FELs [12] such as FLASH [13], LCLS [14-16], and the soon coming European XFEL [17] have been particularly impressive over the last decade [13, 18]. Furthermore, the application of pump-probe techniques [19] in combination with these sources allows for novel and unique time-dependent investigations practically in the femto- [20-22] and more recently even attosecond [23] time domain. Time-domain X-ray spectroscopy techniques are drawing attention from a variety of scientific fields due to their potential to reveal details of fast dynamic processes in matter. The prospects to probe changes in local electronic structures and chemical transformations in real time are a major force in the development of laboratory-based [2023] and accelerator-based [11, 12, 14-16] ultrafast X-ray techniques that complement both energy-domain X-ray spectroscopy efforts at synchrotrons as well as time-domain spectroscopy techniques using ultraviolet, visible, and infrared lasers.

Motivation for the computation of flight times in HDAs has been provided by various publications as, for example, Imhof et al. [24], Caprari [25], Kugeler et al. [26], and Shavorskiy et al. [8]. In these studies the transit time is calculated using an approximate model, which ignores fringing fields at the boundaries. Suggestions to improve the time resolution in coincidence experiments have been made by Volkel and Sandner [10]. An extensive study of HDAs employing elliptical trajectories applicable also to paracentric HDAs including basic timing properties has been presented by Zouros and Benis [1].

Biased paracentric HDAs represent a novel class of HDAs, which use the lensing action of the strong fringing fields at the HDA entry, to restore the 1st-order focus characteristics of the ideal HDAs in a controlled way. The use of this arrangement was first motivated by the work of $[27,28]$, who measured the energy distribution of electrons using an HDA with a mean radius $\bar{R}=101.6 \mathrm{~mm}$, but with an entry displaced from the mean radius (i.e., paracentric) at $R_{0}=$ $82.55 \mathrm{~mm}$. The advantage of using this type of unconventional paracentric configuration is that the effects of angular aberrations on the energy dispersion are minimized. This improvement in energy resolution (and transmission) is attained without the use of any additional fringing field correction electrode schemes and therefore is of particular interest to analyzers using position sensitive detectors as most modern HDAs do nowadays. Measurements using such a paracentric HDA in the Auger electron spectroscopy of projectile ions in collisions with gas targets at the $7 \mathrm{MV}$ tandem accelerator of Kansas State University (KSU) have been reported since 1998 [28]. Recently, the first differential cross sections measurements for electron scattering have also been performed using a biased paracentric system [29]. The biased paracentric HDA from KSU is presently in operation in the APAPES project $[30,31]$ for zero-degree Auger projectile electron spectroscopy at the new atomic physics experimental station located at the $5 \mathrm{MV}$ tandem accelerator at the NCSR Demokritos in Athens.

Methods of optimizing the intrinsic fringing fields to restore 1st-order focusing and improve energy resolution are given in a number of articles [32-35]. However, to the best of our knowledge, no systematic studies on the transit time spread of biased paracentric HDAs exist to date. In this work, we calculate the transit time and energy distributions of electrons within the biased paracentric HDAs for two paracentric entry positions $R_{0}=82.55 \mathrm{~mm}$ and $R_{0}=116 \mathrm{~mm}$ and compare them to those for entry $R_{0}=\bar{R}=101.6 \mathrm{~mm}$ (the conventional HDA mean radius entry) using the computer-based finite-difference trajectory simulation software SIMION 8.1 [36]. The particular values of $R_{0}$ in the two paracentric entry positions were discovered by previous systematic numerical search also using SIMION [34]. In addition, other such paracentric positions for a variety of HDAs with different mean radii $\bar{R}$ and interradial separations $\Delta R$ were also presented in a previous systematic investigation [33].

Here, we present simulations of spreads in both exit position and time as a function of entry aperture size $\Delta r_{0}$ (typically controlled by the magnification of the injection lens), the HDA launching angle spread $\alpha_{\max }$, and the spread in energy $\delta E$ at the fixed pass energy of $E_{0}=1 \mathrm{keV}$. The transit time and energy spreads are also reported for pass energies $E_{0}=10,100,500 \mathrm{eV}$ for particular practical initial conditions. In Section 2, the theoretical background is summarized based on the Kepler orbit equation of an ideal field HDA [1] and new results both in the time spreads and also in the radial spreads are presented in closed form analytic results (where possible). These results are then verified using SIMION simulations both for the ideal field and also for the fringing field HDA. In Section 3, the SIMION simulation procedure is described and in Section 4 our results are presented for both fringing and ideal field HDAs and discussed. Comparisons of both paracentric and conventional entry results are made and a figure of merit is obtained for the best combination of energy and transit time distributions.

\section{Theoretical Background}

The analytical solution of the equation of motion in the ideal $1 / r$ field of an HDA is well documented in the extensive literature (see $[1,4,5,37-40]$ and references therein). In particular, [1] developed in detail the theoretical framework for analyzing the elliptical (Kepler) trajectories of charged particles in the ideal HDA and is extensively referenced. In this section, we only give a summary of the necessary theoretical results and present the final working equations for the energy resolution and the time-of-flight of electrons in an ideal HDA. These equations are then used as a benchmark for the detailed intercomparison of the time-of-flight and energy resolution properties of the ideal HDA to those of the "real" (fringing field) HDA whose simulation in SIMION is presented in Sections 3 and 4. 


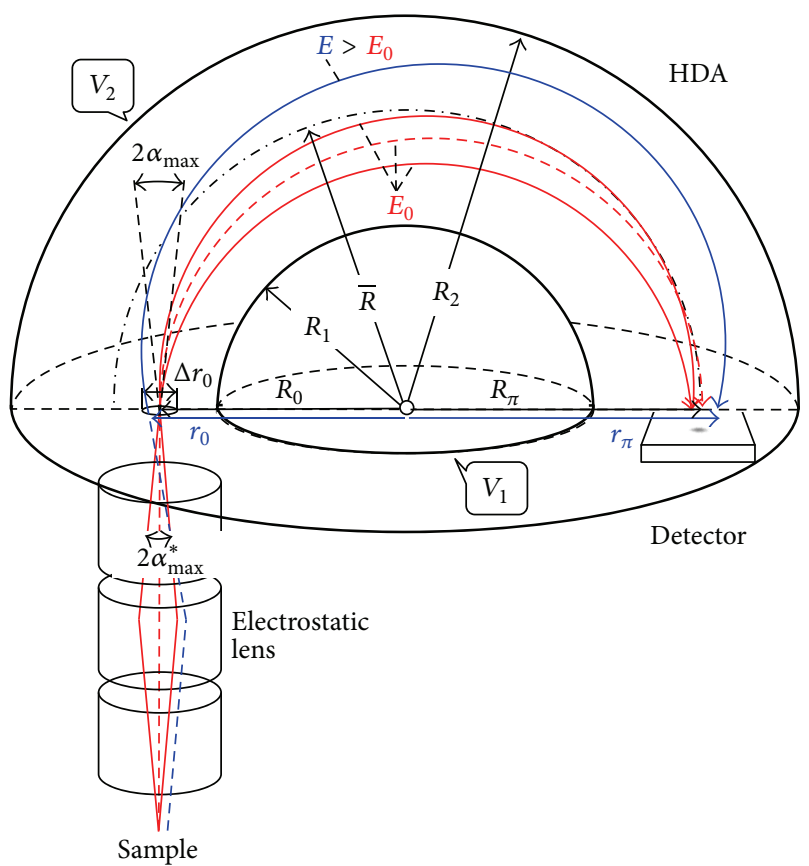

FIGURE 1: HDA schematic consisting of two concentric hemispheres with radii $R_{1}$ and $R_{2}$, an electrostatic lens at input, and a position sensitive detector at the exit. The HDA voltages are set to pass the reference trajectory (red dashed line) having nominal pass energy $E_{0}$ and launching angle $\alpha=0$, with specified entry radius $R_{0}$ and exit radius $R_{\pi}$. Two additional trajectories (red lines) of the same energy $E_{0}$ are shown to originate from a point source, but with $\alpha=$ $\pm \alpha_{\max }$. Here, the entry is seen to be paracentric with $R_{0}$ displaced from $\bar{R}$, the mean radius. The dash-dot line shows the conventional optical axis at $\bar{R}$ for central entry $R_{0}=\bar{R}$. A general trajectory is also shown (in blue) with pass energy $E$ seen to start at entry radius $r_{0}$ and launching angle $\alpha$, exiting the HDA at radius $r_{\pi}$. The PSD records the exit positions of the trajectories.

As shown in Figure 1, the generic HDA consists of two concentric hemispherical electrodes of radii $R_{1}$ and $R_{2}$ $\left(R_{1}<R_{2}\right)$. Typically, electrons enter the HDA with an energy $E$, an angular spread $\pm \alpha_{\max }$ (with respect to the optical axis), and a spatial spread $\Delta r_{0}$ around the entry radius $r_{0}$. After a deflection through $180^{\circ}$ in the HDA field electrons exit at radius $r_{\pi}$ after a transit time $t_{\pi}$ and are recorded by a detector. The angular $\left( \pm \alpha_{\max }\right)$ and spatial $\left(\Delta r_{0}\right)$ entry spreads and energy spreads $\delta E$ (for a nonmonoenergetic source) contribute to the overall energy and transit time spreads $\Delta E$ and $\Delta t$, recorded at the HDA exit. In addition, most modern HDAs are typically equipped with a cylindrical electrostatic input lens whose symmetry axis is placed at $R_{0}$ and a $1 \mathrm{D}$ or $2 \mathrm{D}$ position sensitive detector (PSD) at the exit. The lens system is used to collimate and focus the source emission onto the entry plane of the HDA and will not be considered in this investigation. The PSD offers multichannel detection capability decreasing substantially the data collection time since in this case the analyzer energy need not be scanned to record a spectrum. 2D capabilities may offer useful additional diagnostics or important angular information.
2.1. Kepler Orbits and General Radial Orbit Equation. For motion in the $1 / r$ potential of an ideal HDA given by

$$
V(r)=-\frac{k}{r}+c,
$$

the nonrelativistic trajectory of a particle with charge $q(q=$ $-e$ for electrons), kinetic energy $E$, and angular momentum $L$ is given by (see equation (98) in [1])

$$
r(\omega)=\frac{p}{1+\epsilon \cos \left(\omega-\omega_{\epsilon}\right)} .
$$

The particle enters at radius $r_{0}=r(\omega=0)$, with launching angle $\alpha$ within the entry aperture of diameter $\Delta r_{0}$ centered at $R_{0}$. It then follows in general an elliptical trajectory $r(\omega)$ given as a function of the orbit angle $\omega$ and exits at $r_{\pi}=r\left(\omega=180^{\circ}\right)$ after going through an orbit of $\pi\left(\Delta \omega=180^{\circ}\right)$. The central trajectory (red dashed line in Figure 1) used as reference is defined as the trajectory of a particle which enters with energy $E_{0}$ at $r_{0}=R_{0}$ with $\alpha=0^{\circ}$ and exits at $r_{\pi}=R_{\pi}$. At the exit either a slit or an aperture is typically placed with center at $R_{\pi}$ or alternatively a PSD is used. The conventional HDA arrangement typically utilizes $R_{0}=R_{\pi}=\bar{R}$ in which case the central trajectory is a circle. By convention [1] the trajectory launched with $\alpha>0$ is always an outer trajectory, while the one with $\alpha<0$ is always an inner trajectory.

The parameters $p$ and $\epsilon$ in (2) are the latus rectum and eccentricity of the resulting elliptical trajectory given by [1]

$$
\begin{aligned}
& p=\frac{L^{2}}{m q k}, \\
& \epsilon=\sqrt{1+\frac{2 E L^{2}}{m q^{2} k^{2}}}
\end{aligned}
$$

with the kinetic energy $E$ and (vector) angular momentum $\mathbf{L}$ inside the HDA given by

$$
\begin{aligned}
& E=\frac{1}{2} m v^{2}, \\
& \mathbf{L}=\mathbf{r} \times m \mathbf{v},
\end{aligned}
$$

where $\mathbf{v}$ is the velocity of the particle at radius $r$.

The potential constants $k$ and $c$ given in (1) are fixed by the voltages $V_{1}$ and $V_{2}$ supplied to the hemispherical electrodes and do not depend on the trajectory parameters of the arbitrary particle. These voltages are determined by the central trajectory (already defined above) and are discussed later in this section (see (10)). The trajectory described by (2) is in general part of an ellipse with one focus at the center of force $r=0$ with the constant $\omega_{\epsilon}$ set by the initial conditions. The shape of the elliptical orbit is well defined by $p$ and $\epsilon$ which are constants of the motion, since they are determined from the particle's total energy $E_{\text {tot }}=E-q c$ (see equation (74) in [1]) and vector angular momentum $\mathbf{L}$, both conserved quantities for motion in any purely radial potential $V(\mathbf{r})=V(r)$. We note that, in 
the fringing field HDA, even though the potential is not radial $[V(\mathbf{r})=V(r, \theta)$; i.e., there is an additional dependence on the polar angle $\theta$ ] and therefore the magnitude of the angular momentum $L=|\mathbf{L}|$ is not conserved, the direction of the angular momentum $\mathbf{L} / L$ is conserved and therefore motion remains planar.

Assuming that the particle enters the HDA at time $t=\omega=$ 0 with entry radius $r_{0}=r(\omega=0)$ and launching angle $\alpha$ and then exits at orbit angle $\omega=\pi$ with exit radius $r_{\pi}=r(\omega=\pi)$ at $t=t_{\pi}$, the latus rectum $p$ and the eccentricity $\epsilon$ of the ellipse as a function of $\alpha$ and $r_{0}$ are given by (see equation (40) of [1])

$$
\begin{aligned}
& p=a \rho_{0}\left(2-\rho_{0}\right) \cos ^{2} \alpha, \\
& \epsilon=\sqrt{\sin ^{2} \alpha+\left(1-\rho_{0}\right)^{2} \cos ^{2} \alpha}
\end{aligned}
$$

with

$$
\begin{aligned}
& \rho_{0} \equiv \frac{r_{0}}{a}, \\
& a \equiv a(E)=\frac{q k}{2(q c-E)},
\end{aligned}
$$

where $a$ is the semimajor axis of the ellipse given in terms of constants $q, k$, and $c$ and the particle kinetic energy $E$. Under the specified initial conditions, the elliptical trajectory can be further specified as

$$
r(\omega)=\frac{p r_{0}}{r_{0}+\left(p-r_{0}\right) \cos \omega-p \sin \omega \tan \alpha}
$$

showing it to have both odd and even (through $p$ given in (6a), (6b), (6c), and (6d)) terms in the launching angle $\alpha$.

The values of the potential constants $k$ and $c$ in (1) are specified so that the central trajectory used as reference enters the HDA at $\omega=0$ with $\alpha=0$ and $r_{0}=R_{0}$ and kinetic energy $E_{0}$ and exits at $\omega=\pi$ with $r_{\pi}=R_{\pi}$. Applying these conditions to (7) we obtain (see equations (86) and (87) of [1])

$$
\begin{aligned}
& q k=\frac{E_{0}\left(R_{0}+R_{\pi}\right) \gamma R_{0}}{R_{\pi}}, \\
& q c=E_{0}\left(1+\frac{\gamma R_{0}}{R_{\pi}}\right) .
\end{aligned}
$$

It is seen from (6a), (6b), (6c), and (6d) and (8a)-(8b) that the reference trajectory has a semimajor axis $a_{0}$ and eccentricity $\epsilon_{0}$ given by

$$
\begin{aligned}
& a_{0} \equiv a\left(E=E_{0}\right)=\frac{1}{2}\left(R_{\pi}+R_{0}\right), \\
& \epsilon_{0} \equiv \epsilon\left(\alpha=0, r_{0}=R_{0}, E=E_{0}\right)=1-\frac{R_{0}}{a_{0}}=\frac{R_{\pi}-R_{0}}{R_{\pi}+R_{0}}
\end{aligned}
$$

which for the centric conventional HDA entry $\left(R_{\pi}=R_{0}=\bar{R}\right.$, $\gamma=1)$ is a circle with $\bar{a}_{0} \equiv \bar{R}$ and $\bar{\epsilon}_{0} \equiv 0$ as expected.

Using (8a)-(8b) and (1) we may next obtain the potential $V(r)$ given by [1]

$$
q V(r)=E_{0}\left\{1-\gamma\left(\frac{R_{0}}{R_{\pi}}\right)\left[\frac{R_{0}+R_{\pi}}{r}-1\right]\right\}
$$

from which the HDA voltages can be immediately obtained:

$$
\begin{array}{r}
q V_{i} \equiv q V\left(R_{i}\right)=E_{0}\left\{1-\gamma\left(\frac{R_{0}}{R_{\pi}}\right)\left[\frac{R_{0}+R_{\pi}}{R_{i}}-1\right]\right\} \\
(i=1,2) .
\end{array}
$$

For the above voltages the general trajectory $r(\omega)$ can then be readily shown to be given by

$$
r(\omega)=\frac{2 r_{0} \cos ^{2} \alpha}{\left[\left(\gamma(1+\xi)-\left(r_{0} / R_{0}\right)(\gamma-\xi(\tau-1)) \cos \omega\right) /\left(\gamma(1+\xi)-\left(r_{0} / R_{0}\right)(\gamma-\xi(\tau-1))\right)\right]+\cos (2 \alpha+\omega)},
$$

where we have also introduced the short hand of the following [1]: $\xi \equiv R_{\pi} / R_{0}$ and $\tau \equiv E / E_{0}$. Also introduced in (8a), (8b), (8c), and (8d)-(11) is the entry bias parameter $\gamma$. Equation (11) is seen to satisfy the conditions of the central trajectory; that is, $r\left(\omega=\pi, \alpha=0, r_{0}=R_{0}, \tau=1\right)=R_{\pi}$ independent of the value of $\gamma$. However, $\gamma$ does affect all other trajectories as seen from (11) and can therefore be used as an additional parameter to optimize the HDA performance. Setting $r_{0}=R_{0}$ and $E=E_{0}$ in (11) we obtain the defining relation [1]:

$$
\gamma \equiv 1-\frac{q V_{0}}{E_{0}}
$$

where $V_{0} \equiv V\left(R_{0}\right)$ is the value of the potential at the entry $R_{0}$. We note that when $\gamma=1, V_{0}=0$ and vice versa. Since the particle's effective total energy $E_{\text {tot }}$ inside the HDA must always be negative for bound motion we must always have $E_{\text {tot }}=E-E_{0}(1+\gamma / \xi)<0$ which leads to the restriction on $\gamma$ :

$$
\gamma>\xi(\tau-1)
$$

which is also consistent with the requirement that the semimajor axis $a>0$ since using (8a)-(8b) $a$ from (6d) can be expressed as

$$
a=a(\tau)=\frac{R_{0}(1+\xi)}{2[\xi(1-\tau)+\gamma]} .
$$

In the past, we have reported on the properties of HDAs and their dependence on the entry parameters $\xi$ and $\gamma$. 
Quite generally, we can identify two different entry classes as follows:

(i) the conventional entry HDA for which $R_{0}=\bar{R}=R_{\pi}$, that is, $\xi=1$, and whose reference trajectory is a circle $(\epsilon=0)$;

(ii) the paracentric entry HDA for which $\xi \neq 1$, whose reference trajectory is an ellipse $(0<\epsilon<1)$.

For both types of entry, $\gamma$ can be independently used to bias the entry. Thus, for conventional HDAs, zero bias is typically used having $V_{0}=0(\gamma=1)$. For paracentric entries, however, both positive bias $\left(V_{0}>0\right.$ or $\left.\gamma>1\right)$ and negative bias $\left(V_{0}<0\right.$ or $\left.\gamma<1\right)$ have been investigated $[1,7,27,29,33,34]$. While the value of $\gamma$ in an ideal HDA does not affect its overall 1st-order focusing, in a real fringing field HDA, for which the conventional entry is well known to lose 1st-order focusing, specific combinations of optimal $(\xi, \gamma)$ values have been found to restore 1st-order focusing [29, 33-35]. In Section 4, we will look at a concrete example of such an HDA having $R_{1}=72.4 \mathrm{~mm}$ and $R_{2}=132.8 \mathrm{~mm}$ $\left(R_{\pi}=\bar{R}=101.6 \mathrm{~mm}\right)$ and investigate both the energy and the time resolution for paracentric entry $R_{0}=82.55 \mathrm{~mm}$ with $\gamma=1.4715$ and $R_{0}=116 \mathrm{~mm}$ with $\gamma=0.5$ in comparison to the conventional HDA with $R_{0}=\bar{R}$ and $\gamma=1$. These particular combinations of $(\xi, \gamma)$ have been found [29, 33-35] to date, to restore 1st-order focusing. Such an HDA is being used in our laboratory for high-resolution Auger projectile electron spectroscopy [7, 27-29, 31, 35] with excellent energy resolution. In [33], we have also investigated numerically HDAs with different values of $R_{1}$ and $R_{2}$ and have also found corresponding optimal combinations of $(\xi, \gamma)$ which restore 1st-order focusing.

2.2. HDA Exit Radius $r_{\pi}$, Radial Spread $\Delta r_{\pi}$, and Base Energy Resolution $\Delta E_{B}$. Using (11), the exit radius $r_{\pi}=r(\omega=\pi)$ can be computed (see equation (99) and correction of equation (100) in Erratum of [1]) and is given by

$$
\begin{aligned}
r_{\pi} & \equiv r_{\pi}\left(r_{0}, \tau, \alpha, \gamma\right)=-r_{0} \\
& +\frac{r_{0}}{1+\cos ^{2} \alpha\left\{\left(r_{0} /\left(R_{0}+R_{\pi}\right)\right)[1+(\xi / \gamma)(1-\tau)]-1\right\}}
\end{aligned}
$$

which may also be expanded in powers of $\alpha$ giving up to 5th order

$$
\begin{aligned}
\frac{r_{\pi}}{r_{0}} & =-1+\frac{\Delta}{r_{0}}\left\{1-\alpha^{2}\left(1+\frac{\Delta}{r_{0}}\right)\right. \\
& \left.+\alpha^{4}\left[\frac{2}{3}-\frac{\Delta}{r_{0}}\left(\frac{5}{3}-\frac{\Delta}{r_{0}}\right)\right]\right\}+O\left[\alpha^{6}\right]
\end{aligned}
$$

with

$$
\Delta^{2} \equiv \frac{D_{\gamma}\left(R_{0}+R_{\pi}\right) \gamma}{\xi \tau}
$$

and where it is readily seen that

$$
r_{\pi}\left(r_{0}, \tau, \alpha=0, \gamma\right)=\Delta-r_{0}=\frac{R_{0}+R_{\pi}}{1+(\xi / \gamma)(1-\tau)}-r_{0}
$$

The energy dispersion $D_{\gamma}$ is another useful analyzer parameter defined in general as

$$
\begin{aligned}
D_{\gamma} & \equiv E\left(\frac{\partial r_{\pi}}{\partial E}\right)_{\alpha=0}=D_{\gamma}(\tau) \\
& =\frac{(1+\xi) R_{\pi}}{\gamma[1+(\xi / \gamma)(1-\tau)]^{2}} \tau .
\end{aligned}
$$

For slit HDAs, where $\tau=1$ (i.e., $E=E_{0}$ ), $D_{\gamma}$ is a characteristic length of the HDA which determines the shift of the image position of a particle trajectory for an infinitesimal change in the particle energy $E_{0}$ [5]. However, for HDAs with PSD its definition can be extended to any $E$ as above. Equation (14) is also known as the basic equation of the analyzer [1]. As can be verified in (14), the reference trajectory $\left(r_{0}=R_{0}, \tau=1\right.$, $\alpha=0)$ always exits at $R_{\pi}$. We note that a similar equation can also be expressed in terms of $\alpha^{*}$, the entry angle into the HDA (see equation (102) in [1] and Figure 1). The angles $\alpha$ and $\alpha^{*}$ are related according to Snell's law [1]. Here, our treatment is given in terms of the angle $\alpha$ inside the HDA, which is the launching angle typically used in simulations. A more complete treatment including the effect of the injection lens should be expressed in terms of the outside (incident) entry angle $\alpha^{*}$ as determined by the lens and thus also include the effect of refraction due to the potential change at $r_{0}$ in crossing the HDA entry plane. Because typically HDA entry apertures are small we in general have $V\left(r_{0}\right) \approx V\left(R_{0}\right)$ and therefore for conventional HDAs which use $\gamma=1, V\left(r_{0}\right) \approx 0$ and refraction can be neglected. This clearly is not the case for paracentric entry where optimum values of $\gamma$ are typically different from 1 and therefore $V\left(r_{0}\right) \neq 0$.

It is also obvious from (14) that only even powers of $\alpha$ are expected and therefore we should have

$$
r_{\pi}(\alpha)=r_{\pi}(-\alpha)
$$

indicating 1st-order focusing, a basic requirement for any energy analyzer. For monoenergetic beams of particles with energy $E$ we may vary the input beam parameters $\Delta r_{0}$ and $\alpha$ to obtain the maximum allowed exit radius variation $\Delta r_{\pi}$ [41]. At the HDA exit plane this can be shown [7, 41] to be equivalent to the following calculation:

$$
\begin{aligned}
\Delta r_{\pi}= & r_{\pi}\left(R_{0}-\frac{\Delta r_{0}}{2}, E, \alpha=0\right) \\
& -r_{\pi}\left(R_{0}+\frac{\Delta r_{0}}{2}, E, \alpha_{\max }\right)
\end{aligned}
$$


yielding

$$
\begin{aligned}
\Delta r_{\pi} & =\Delta r_{0}+\frac{\left(R_{0}+R_{\pi}\right)}{1+(\xi / \gamma)(1-\tau)} \\
- & \frac{\left(1+\Delta r_{0} / 2 R_{0}\right)\left(R_{0}+R_{\pi}\right)}{(1+\xi)-\left[\left(\xi-\Delta r_{0} / 2 R_{0}\right)-\left(1+\Delta r_{0} / 2 R_{0}\right)(\xi / \gamma)(1-\tau)\right] \cos ^{2} \alpha}
\end{aligned}
$$

which may be expanded in powers of $\Delta r_{0}$ and $\alpha_{\max }$ to give

$$
\begin{aligned}
\Delta r_{\pi}= & \sum_{i=0, j=0} P_{i j}\left(\alpha_{\max }\right)^{i}\left(\Delta r_{0}\right)^{j} \\
= & P_{01} \Delta r_{0}+P_{10} \alpha_{\max }+P_{20} a_{\max }^{2}+P_{21} a_{\max }^{2} \Delta r_{0} \\
& +O[4]
\end{aligned}
$$

where

$$
\begin{aligned}
P_{01} & =|M|=1, \\
P_{00}=P_{10} & =P_{11}=0, \\
P_{20} & =D_{\gamma}\left(\frac{\tau+\gamma-1}{\tau}\right), \\
P_{21} & =-\frac{\gamma(1+\xi)}{\xi \tau}\left(\frac{D_{\gamma}}{R_{0}}\right) .
\end{aligned}
$$

Here, $P_{10}$ and $P_{20}$ are the well-known 1st- and 2nd-order $\alpha$ angular aberration coefficients, respectively, seen to depend on $\tau$ in the case of an HDA with PSD. For a slit HDA, however, we have $\tau=1$ and these are characteristics of the analyzer. Finally, $M$ is the analyzer magnification with $M=-1$ for an ideal HDA. For most analyzers $P_{10}=0$, the well-known 1storder focusing condition. For a conventional $(\xi=\gamma=1$, $\left.R_{0}=\bar{R}\right)$ HDA we obtain the well-known [5] case $P_{20}=2 \bar{R}$.

For most of electron spectroscopy and spectroscopic imaging applications, the relative energy resolution of an HDA, $\Delta E / E$, is an important consideration; see [1-5, 42-45] and references therein. The absolute base energy resolution $\Delta E_{B}$, defined as "the range of energies over which a monoenergetic beam produces an output in an analyzer set at fixed deflection voltage," can be related to the exit width $\Delta r_{\pi}$ by [5]

$$
\frac{\Delta E_{B}}{E}=\frac{\delta r_{\pi}+\Delta r_{\pi}}{D_{\gamma}}
$$

where $\delta r_{\pi}$ is the spatial resolution of the PSD (or the exit slit width) in the dispersion direction, while $\Delta r_{\pi}$ is the maximal position spread at the exit for a monoenergetic beam of energy $E$. Using the results of (20) in (22) and keeping only up to 2ndorder terms, we finally obtain the well-known formula for the relative base energy resolution of an ideal HDA:

$$
\frac{\Delta E_{B}}{E}=\frac{\left(\Delta r_{0}+\delta r_{\pi}\right)}{D_{\gamma}}+\left[1+\frac{(\gamma-1)}{E / E_{0}}\right] a_{\max }^{2} .
$$

Equation (23) is particularly applicable to HDAs using a PSD for which in general $E$ is different from the reference energy $E_{0}$. For any ideal HDA with slits/apertures we need $E=E_{0}$ and find $P_{10}=0, P_{20}=\gamma D_{\gamma}, M=-1$ with $D_{\gamma}$ now given by (16) with $\tau=1\left(E=E_{0}\right)$ :

$$
\left.D_{\gamma}(\tau=1)=\left(R_{0}+R_{\pi}\right) \frac{R_{\pi}}{\gamma R_{0}} \quad \text { (Slit HDA }\right)
$$

for which we then also have

$$
\frac{\Delta E_{B}}{E_{0}}=\gamma\left(\frac{\Delta r_{0}+\delta r_{\pi}}{R_{0}+R_{\pi}}\right)\left(\frac{R_{0}}{R_{\pi}}\right)+\gamma \alpha_{\max }^{2}
$$

(Slit HDA).

Finally, for the ideal conventional $\operatorname{HDA}\left(R_{0}=R_{\pi}=\bar{R}, \gamma=1\right.$, $\left.E=E_{0}\right)$ we obtain the well-known result:

$$
\frac{\Delta E_{B}}{E_{0}}=\left(\frac{\Delta r_{0}+\delta r_{\pi}}{2 \bar{R}}\right)+\alpha_{\max }^{2}
$$

(Conventional Slit HDA).

In real applications, ideal energy resolution formula equations (23), (25), and (26) are only used as a general guide, since it is well known that, without any additional fringing field correction, the real HDA has a substantial nonzero coefficient $P_{10}$ and thus is no more 1st-order focusing [34]. Note, however, that the HDA electrode voltages are still typically specified by the ideal HDA voltages given by (10).

Over the last 15 years, the authors and their collaborators have shown [27-30, 32-35] that the biased paracentric HDA does in fact overcome the fringing field problem without the need to introduce any additional electrodes [33, 34]. This is done by moving the entry point $R_{0}$ to the so-called paracentric position and optimizing the entry bias $\gamma$ so that the lensing action of the fringing fields restores 1st-order focusing conditions. For the present HDA these paracentric positions have been found through simulation to be at $R_{0}=$ $82.55 \mathrm{~mm}$ with $\gamma=1.4715$ and $R_{0}=116.0 \mathrm{~mm}$ with $\gamma=$ 0.499 [34]. In general, while paracentric entries are thought to exist for any fringing field HDA (particularly for those using a large interradial distance in order to accommodate a PSD), no general scaling rules [34] have been discovered to date so their exact values have to be found by simulation in combination with the corresponding optimum value of $\gamma$.

Very recently, the improvement of the energy resolution for the above paracentric entries found in simulation was shown to be valid also in experiment [29]. However, to date, there have been no investigations of the timing properties of paracentric entries. This is explored next in Section 2.3 for the ideal HDA and in Sections 3 and 4 for the fringing field via simulations using the charged particle optics code SIMION [36].

2.3. Time-of-Flight $t_{\pi}$ and HDA Exit Time Spread $\Delta t_{\pi}$. As shown in Figure 1, electrons entering the HDA with launching angles $\alpha$ follow elliptical trajectories in the radial electrostatic field. Due to the different initial conditions of their trajectories, a spread in their transit times accrues. Neglecting the fringing fields at the boundaries and other mechanical imperfections, the transit time, $t_{\pi}$, of a particle 
with mass $m$ and charge $q$ through an orbit of $\Delta \omega=\pi$ in the ideal HDA is computed following the treatment in [1]. Observing the rotational velocity to be, $v_{\omega}=r \dot{\omega}=$ $L / m r$, where $L$ is the conserved angular momentum, we then have

$$
t_{\pi}=\int_{0}^{\pi} \frac{m r^{2}(\omega)}{L} d \omega .
$$

Using (3)-(4) we can directly integrate (27) to obtain (using Wolfram Mathematica 9)

$$
\begin{aligned}
& t_{\pi}\left(\alpha, a, \rho_{0}\right)=\frac{T_{0}}{2 \pi}\{\pi \\
& +2 \operatorname{ArcTan}\left[\sin \alpha \sqrt{\left(\frac{2}{\rho_{0}}-1\right)}\right] \\
& \left.-\frac{4\left(\sqrt{\rho_{0} /\left(2-\rho_{0}\right)}\right) \sin \alpha}{\left[\cos 2 \alpha-\left(\left(2+\rho_{0}\right) /\left(2-\rho_{0}\right)\right)\right]}\right\},
\end{aligned}
$$

where $T_{0}$ is the period of the full Kepler orbit given by

$$
\begin{aligned}
T_{0} & =2 \pi \sqrt{\frac{m a^{3}}{q k}}=\left[\frac{\pi R_{0}(1+\xi) \gamma \sqrt{\xi}}{(\gamma+\xi(1-\tau))^{3 / 2}}\right] \sqrt{\frac{m}{2 E_{0}}} \\
& =\pi \gamma^{2}\left(\frac{D_{\gamma}}{\tau}\right) \sqrt{\frac{\gamma+\xi(1-\tau)}{\xi}} \sqrt{\frac{m}{2 E_{0}}}
\end{aligned}
$$

which for the ideal centric conventional HDA entry $\left(R_{\pi}=\right.$ $R_{0}=\bar{R}, \gamma=1$ ) becomes

$$
\bar{T}_{0} \equiv 2 \pi \bar{R} \sqrt{\frac{m}{2 E_{0}}} .
$$

It is practical to use $m=5.68569 \mathrm{eV}-\mathrm{ns} / \mathrm{mm}$ for the electron mass so that when used with kinetic energy $E_{0}$ in $\mathrm{eV}$ and distances in $\mathrm{mm}$ timing results are directly obtained in $\mathrm{ns}$. We have done so in all our timing calculations in this paper. We note that expressions for $t_{\pi}$ are also given in $[25,26]$, but (28) seems to be simpler and is found to be in agreement both with [25] and also with nonrelativistic SIMION for motion in the ideal field HDA.

Equation (28) may also be expanded in powers of $\alpha$ as shown in [1]. However, the expression given there (equation (56) of [1]) has an error in the $\alpha^{5}$ term. Here we have performed again the evaluation (using Wolfram Mathematica 9) and obtained the new corrected result:

$$
\begin{aligned}
t_{\pi} & =\frac{T_{0}}{2 \pi}\left\{\pi+\sqrt{\left(\frac{2}{\rho_{0}}-1\right)}\left[4 \alpha-2\left(\frac{8}{3 \rho_{0}}-1\right) \alpha^{3}\right.\right. \\
& \left.\left.+\left(33-\frac{208}{\rho_{0}}+\frac{288}{\rho_{0}^{2}}\right) \frac{\alpha^{5}}{30}+O\left[\alpha^{7}\right]\right]\right\} .
\end{aligned}
$$

As can be seen clearly now from (30), the time-of-flight of a trajectory starting with negative launching angle $\alpha<0$ will in general be smaller than that with $\alpha>0$. This is primarily because the trajectory for $\alpha<0$ will always be shorter. For $\alpha=0,(28)$ and (30) both give the correct half period $T_{0} / 2$. Furthermore, as known from [1] and also as discerned from Figure 2 we also have for the ideal field

$$
t_{\pi}(\alpha)+t_{\pi}(-\alpha)=T_{0}=2 t_{\pi}(0)
$$

which again is seen to be in agreement with the results of (28) and (30).

Using (28) we investigate the spread in the transit time $\Delta t_{\pi}$ for a monoenergetic beam of energy $E$ for corresponding spreads in $\alpha$ and $r_{0}$ as we did in the case of $r_{\pi}$. In this case the maximal transit variation can be seen to be given by

$$
\begin{aligned}
\Delta t_{\pi} \equiv & t_{\pi}\left(R_{0}+\frac{\Delta r_{0}}{2}, E, \alpha_{\max }\right) \\
& -t_{\pi}\left(R_{0}-\frac{\Delta r_{0}}{2}, E,-\alpha_{\max }\right),
\end{aligned}
$$

in accordance with the fact that $t_{\pi}$ now depends on odd powers of $\alpha$.

Keeping only up to 3rd-order terms in the series expansion we obtain (using Wolfram Mathematica 9)

$$
\begin{aligned}
\Delta t_{\pi}= & \sum_{i=0, j=0} P_{i j}^{\prime}\left(\alpha_{\max }\right)^{i}\left(\Delta r_{0}\right)^{j} \\
= & P_{10}^{\prime} \alpha_{\max }+P_{11}^{\prime} \alpha_{\max } \Delta r_{0}+P_{20}^{\prime} a_{\max }^{2}+P_{30}^{\prime} a_{\max }^{3} \\
& +O[4]
\end{aligned}
$$

with

$$
\begin{aligned}
P_{00}^{\prime} & =P_{01}^{\prime}=P_{11}^{\prime}=P_{20}^{\prime}, \\
P_{10}^{\prime} & =\left[4 \frac{\sqrt{\gamma+\tau-1}}{\tau}\right] D_{\gamma} \sqrt{\frac{m}{2 E_{0}}} \\
& =\left(\frac{4}{\sqrt{\gamma+\tau-1}} \sqrt{\frac{m}{2 E_{0}}}\right) P_{20}, \\
P_{30}^{\prime} & =-\left[\frac{(\gamma(1+4 \xi)-3 \xi(1-\tau))}{6(\gamma+\xi(1-\tau))}\right] P_{10}^{\prime},
\end{aligned}
$$

and using (29a) and (29b) we can obtain an equation similar to that for $\Delta r_{\pi}$ :

$$
\begin{aligned}
& \frac{\Delta t_{\pi}}{\left[\left(T_{0} / \pi \gamma^{2}\right) \sqrt{\xi(\gamma+\tau-1) /(\gamma+\xi(1-\tau))}\right]} \\
& =2 \alpha_{\max }-\frac{1}{3}\left[\frac{\gamma(1+4 \xi)-3 \xi(1-\tau)}{\gamma+\xi(1-\tau)}\right] a_{\max }^{3} \\
& +O[4] .
\end{aligned}
$$




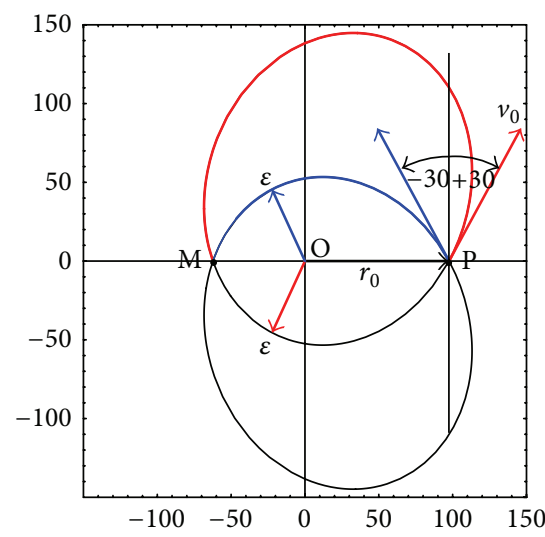

(a)

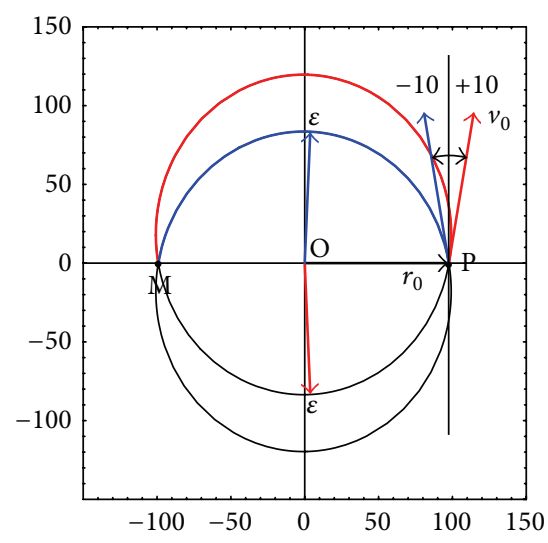

(b)

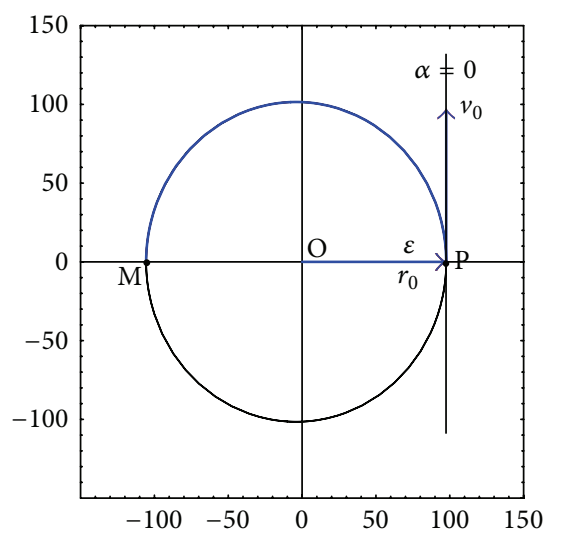

(c)

FIGURE 2: Elliptical orbits with the same kinetic energy $E=E_{0}$ for $\alpha= \pm 30^{\circ}$ (a), $\alpha= \pm 10^{\circ}$ (b), and $\alpha=0^{\circ}$ (c) with $\alpha>0$ (red) and $\alpha<0$ (blue). The field is central with center at $\mathrm{O}$, one of the foci of the ellipse. The electron enters at the point $\mathrm{P}$ with radius $r_{0}$ and angle $\alpha$ and exits at point $\mathrm{M}$ after going through a full $180^{\circ}$. All orbits with $\alpha>0$ are always outer and therefore are always longer in both space and time with corresponding eccentricity vector $\epsilon$ lying in the opposite lower half plane. As $\alpha$ decreases the corresponding eccentricity vectors rapidly rotate towards the positive $x$-axis coalescing for $\alpha=0$, in which case the orbits exactly overlap. The angular momentum $L$ and eccentricity $\epsilon$ are even functions of $\alpha$ and are conserved in the ideal field. Therefore orbits with the same $|\alpha|$ have identical shape but a slightly different, but symmetric, tilt around the nodal line POM as shown. Due to this symmetry orbits with $\alpha>0$ and $\alpha<0$ are complementary giving rise to the condition of (31).

It is interesting to note that the lowest order dependence of $\Delta t_{\pi}$ on $\alpha$ is 1st-order, compared to the 2nd-order $\alpha$ dependence of $\Delta r_{\pi}$ (equations (19)-(20)), thus possibly making it less susceptible to fringing field effects which seem to influence strongly the 1st-order dependence in $\alpha$ (destroy 1storder focusing!) of $\Delta r_{\pi}$. Furthermore, the dependence of $\Delta t_{\pi}$ on $\Delta r_{0}$ is seen to be of higher order in $\Delta t_{\pi}$ but correlated to the value of $\alpha$, again showing $\Delta t_{\pi}$ to be less sensitive to this parameter than $\Delta r_{\pi}$. This behavior is clearly demonstrated in the results of Figure 3.
Finally, a quantity related to the transit time $t_{\pi}$ is the length of the trajectory $s_{\pi}$ given by

$$
\begin{aligned}
s_{\pi} & \equiv \int_{0}^{\pi} d s(\omega)=\int_{0}^{\pi} \sqrt{\left(\frac{d x}{d \omega}\right)^{2}+\left(\frac{d y}{d \omega}\right)^{2}} d \omega \\
& =\int_{0}^{\pi} \sqrt{r(\omega)^{2}+\left(\frac{d r}{d \omega}\right)^{2}} d \omega .
\end{aligned}
$$

This can be simplified (using Wolfram Mathematica 9) to

$$
s_{\pi}\left(\alpha, a, \rho_{0}\right)=\int_{0}^{\pi} \frac{p a \rho_{0} \sqrt{4 a \rho_{0}\left(p-a \rho_{0}\right)(\cos \omega-1)+2 p\left(p \sec ^{2} \alpha-2 a \rho_{0} \sin \omega \tan \alpha\right)}}{\sqrt{2}\left[a \rho_{0}+\left(p-a \rho_{0}\right) \cos \omega-p \sin \omega \tan \alpha\right]^{2}} d \omega .
$$

Equation (36) is seen to include both odd (e.g., $\tan \alpha$ ) and even (due to $p$ from (3a)) powers of $\alpha$ and therefore a condition similar to that of (31) cannot be established also for $s_{\pi}$. Equation (36) can only be evaluated numerically. However, we note that, for $\alpha=0$, further simplifications ensue allowing for the following closed form result (using Wolfram Mathematica 9):

$$
\begin{aligned}
& s_{\pi}(\alpha=0) \\
& \quad=a\left\{\rho_{0} K\left[\frac{4\left(1-\rho_{0}\right)}{\left(2-\rho_{0}\right)^{2}}\right]+\left(2-\rho_{0}\right) E\left[\frac{4\left(1-\rho_{0}\right)}{\left(2-\rho_{0}\right)^{2}}\right]\right\}
\end{aligned}
$$

given in terms of the complete elliptic integral of the first $(K)$ and second $(E)$ kind. The length of the trajectory $s_{\pi}$ can be expected to be longer (shorter) for $\alpha>0(\alpha<0)$, with correspondingly longer (shorter) flight times $t_{\pi}$, respectively.

In the presence of fringing fields, the exit radius $r_{\pi}$, the flight time $t_{\pi}$, and the trajectory length $s_{\pi}$ cannot be established in a simple analytical form. Thus, their calculations have to be carried out numerically. This is done here using the charge particle optics software SIMION [36]. Results are given in Sections 3 and 4, with comparison of ideal and fringing field results. To date, we have shown [33-35] that the fringing field biased paracentric HDA does in fact satisfy (17) to a very good approximation. 


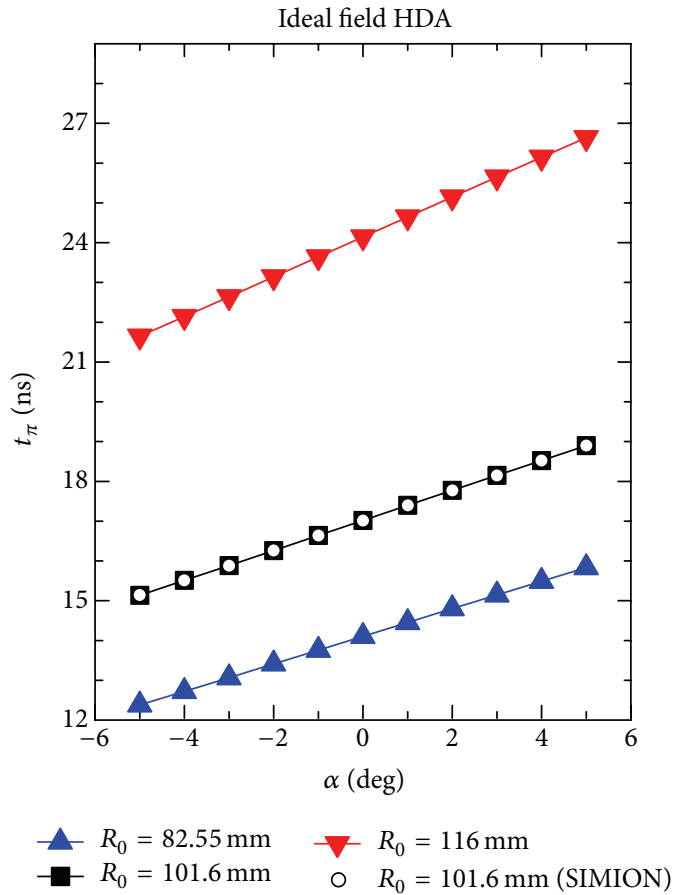

(a)

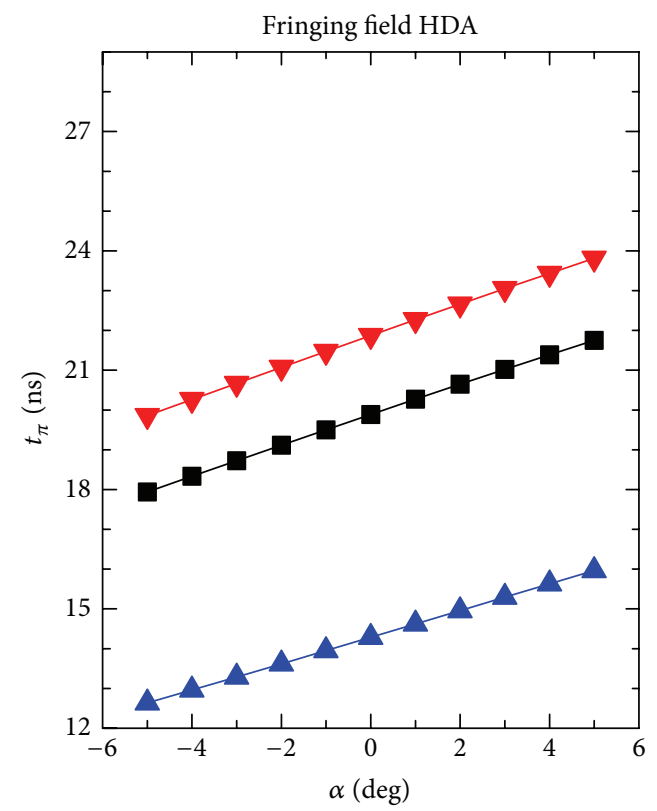

(c)

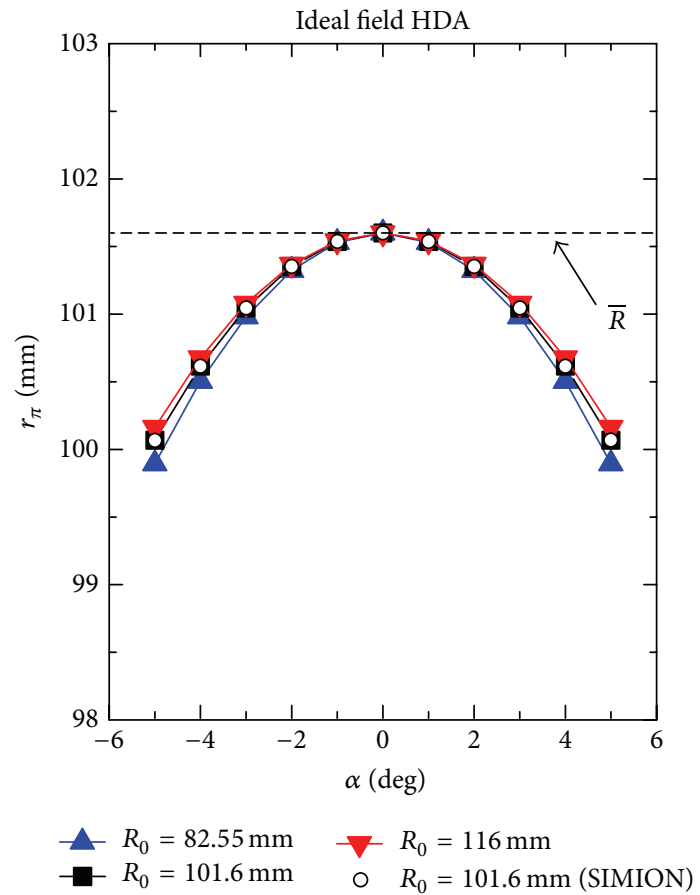

(b)

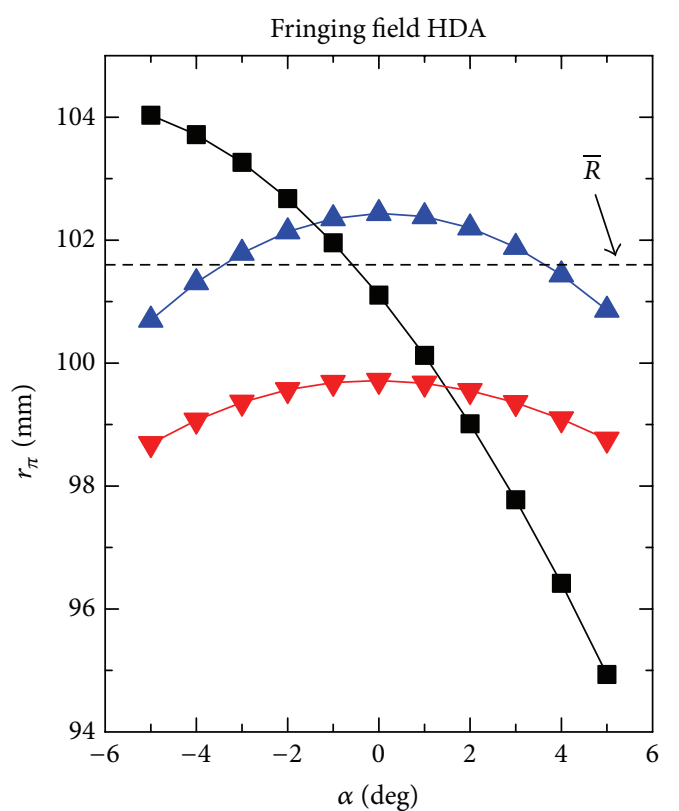

(d)

Figure 3: The time-of-flight $t_{\pi}$ and the exit radius $r_{\pi}$ are plotted as a function of launching angle $\alpha$, for pass energy $E_{0}=1 \mathrm{keV}$ and for a point source $(\Gamma=0)$, in both ideal and fringing field HDAs. In (d), the conventional $\left(R_{0}=\bar{R}=101.6 \mathrm{~mm}\right)$ fringing field HDA (black squares) is seen to have a much larger and asymmetric spread in $r_{\pi}$ as a function of $\alpha$, far from 1st-order focusing conditions. The two paracentric entries $\left(R_{0}=82.55 \mathrm{~mm}\right.$ and $\left.R_{0}=116 \mathrm{~mm}\right)$, however, have much narrower spreads in $r_{\pi}$ demonstrating full 1st-order focusing and therefore much improved energy resolution. The analytical solutions valid for the ideal field HDA in (a) and (b) were obtained using (14) for $r_{\pi}$ and (28) for $t_{\pi}$ 
TABLE 1: HDA parameters with their symbols and values. Voltages $V_{i}$ have been computed from (10).

\begin{tabular}{|c|c|c|c|c|}
\hline Symbol & & Value & & Parameter \\
\hline$R_{1}(\mathrm{~mm})$ & & 72.4 & & Inner HDA radius \\
\hline$R_{2}(\mathrm{~mm})$ & & 130.8 & & Outer HDA radius \\
\hline $\bar{R}(\mathrm{~mm})$ & & 101.6 & & Mean radius \\
\hline$R_{\pi}(\mathrm{mm})$ & & 101.6 & & Exit reference radius \\
\hline$R_{0}(\mathrm{~mm})$ & 82.55 & 101.6 & 116.0 & Paracentric entry radius \\
\hline$\gamma$ & 1.4715 & 1.0 & 0.499 & Entry bias \\
\hline$\xi$ & 1.2308 & 1.0 & 0.8759 & Paracentricity \\
\hline$V_{1}(\mathrm{~V})$ & 845.4089 & 806.6298 & 142.5965 & Inner hemisphere voltage \\
\hline$V_{2}(\mathrm{~V})$ & -512.3477 & -446.4832 & -621.9260 & Outer hemisphere voltage \\
\hline$V_{0}(\mathrm{~V})$ & 471.5 & 0.000 & -501.0 & Nominal entry voltage \\
\hline
\end{tabular}

\section{Calculation Method}

Simulation of the electron motion in electrostatic fields is a subject of growing interest and stimulating research as can be ascertained by the numerous mention of charged particle optics (CPO) software usage at poster exhibitions in conferences. An interesting historical perspective on the evolution of SIMION itself is provided by David Dahl, "father" of SIMION [46], written in 2000. Since 2000, PCs have undergone tremendous improvements in memory, disk space, and speed making CPO programs increasingly more accurate and capable of more sophisticated calculations (see, e.g., [47-49]). Following the theoretical presentation in Section 2, the numerical simulations described in the present work have been realized using the computer program SIMION 8.1, which is a ray-tracing code for threedimensional simulations. SIMION solves the Laplace equation in the HDA for a given geometry utilizing the finitedifference method. This software has been chosen because of its ease in controlling quickly several parameters such as the pass energy, the initial beam parameters (position, pencil angle, etc.), and the voltages of the electrodes. The accuracy of SIMION 7 has been discussed in the past [50] (SIMION 8.1 is much improved) and also been compared to the more accurate BEM approach [33].

In particular, the aim of the transit time calculations is to investigate the suitability of the biased paracentric HDA for simultaneous high energy resolution spectroscopy and time-of-flight measurements within the same HDA. We have chosen the HDA parameters provided by [34]. The three entry positions, $R_{0}=82.55 \mathrm{~mm}, 101.6 \mathrm{~mm}$, and $116 \mathrm{~mm}$, already discussed, were studied. The nominal pass energy was set at $E_{0}=1000 \mathrm{eV}$ for most of the investigations; however at the end of Section 4 we also present results for $E_{0}=10,100,500 \mathrm{eV}$. For each of the three values of $R_{0}$, the HDA voltages depended on both $R_{0}$ and $\gamma$ according to (10). The optimal values of $\gamma$ that achieve the best focusing effect were determined in a previous publication on the specific paracentric HDA: $\gamma \approx 1.5$ for $R_{0}=82.55 \mathrm{~mm}$ and $\gamma=0.5$ for $R_{0}=116 \mathrm{~mm}$ [35]. In Table 1 , we list the values of the most important parameters used in our simulations.

Here, the time resolution is determined by the transit time spread of trajectories passing through the analyzer without the input lens. As in the case of energy resolution, the relative time resolution of the HDA $\left(\Delta t_{\pi} / t_{\pi}\right)$ depends on three main factors: (a) the dimensions such as the diameter of the entry aperture $\Delta r_{0}$, the mean radius $\bar{R}$, and the entry position $R_{0}$, (b) the angular spread of the electron velocity vectors at entry $\left( \pm \alpha_{\max }\right)$, and (c) the input electron beam energy spread $\delta E$ around the pass energy. We assume that the input lens produces an image spot with a Gaussian distribution at the HDA entry plane; the entry beam extent is therefore generally specified in terms of the half width, $\Gamma$, of this distribution. Again we model the energy of the electrons having a Gaussian shape centered around $1 \mathrm{keV}$ and its FWHM is defined by $\delta E$. Our computations cover the ranges $\alpha_{\max } \leq 5^{\circ}, \Gamma \leq$ $1.5 \mathrm{~mm}$, and $\delta E / E_{0} \leq 0.4 \%$, so that the exit position of the electrons lies within the values defined by the detector area. The time spectrum was obtained by ray-tracing calculations where 50,000 electrons were used in each run. Each curve has been normalized to its maximum value.

The lens system at the entry of the HDA (injection lens) can also influence significantly the temporal resolution of the analyzer by modifying the angular spread with which the electrons enter the HDA [8]. According to Liouville's theorem, the volume occupied in phase space by the beam is constant for conservative systems. Thus, the size of the beam can be reduced by the lens but only at the expense of angular divergence [51]. There is also a strong dependence of the electron time spread on the biasing parameter $\gamma$. However, in the present work, this effect was not investigated since $\gamma$ was kept constant at the corresponding values for each of the three entry positions $R_{0}$ shown in Table 1 .

Space charge effects have also been neglected in this work. Other simulation software programs such as CPO, LORENTZ, OPERA, and IBSIMU do have capabilities to handle space charge effects to some extent. Even the newest version of SIMION 8.1 has a Poisson solver and can now also handle some types of space charge problems. For HDAs, space charge effects can be particularly important in cases where the HDA is used as a monochromator [52, 53] (typically to limit the energy width of e-guns, not addressed in this paper) rather than as an analyzer/spectrometer. However, in most cases where the HDA is used as a spectrometer (as in our present treatment) analyzed electron currents 
TABLE 2: Comparison of the electron optical properties for fringing and ideal field cases for each of the three entries computed by SIMION. For all cases, $R_{\pi}=\bar{R}=101.6 \mathrm{~mm}$ and $E_{0}=1 \mathrm{keV}$. Also given in parentheses are the results obtained using the closed form equations (see text). All results are nonrelativistic.

\begin{tabular}{|c|c|c|c|c|c|c|c|}
\hline & & $\begin{array}{l}\text { Po } \\
\text { Pare }\end{array}$ & HDA & Conve & HDA & & $\begin{array}{l}\text { ias } \\
\text { HDA }\end{array}$ \\
\hline & $R_{0}(\mathrm{~mm})$ & & & & & & \\
\hline & $\gamma$ & & & & & & \\
\hline & $V_{0}(\mathrm{~V})$ & & & & & & \\
\hline & & Ideal & Fringing & Ideal & Fringing & Ideal & Fringing \\
\hline & $M$ & $\begin{array}{l}-1.000 \\
(-1.000)\end{array}$ & -0.882 & $\begin{array}{c}-1.000 \\
(-1.000)\end{array}$ & -1.018 & $\begin{array}{c}-1.000 \\
(-1.000)\end{array}$ & -1.500 \\
\hline$r_{\pi}$ & $D_{\gamma}(\mathrm{mm})$ & $\begin{array}{l}154.026 \\
(154.024)\end{array}$ & 159.559 & $\begin{array}{c}203.205 \\
(203.200)\end{array}$ & 206.686 & $\begin{array}{c}381.957 \\
(381.939)\end{array}$ & 320.596 \\
\hline & $P_{10}(\mathrm{~mm} / \mathrm{rad})$ & $\begin{array}{c}0.011 \\
(0.000)\end{array}$ & 0.030 & $\begin{array}{c}0.009 \\
(0.000)\end{array}$ & 106.07 & $\begin{array}{c}0.007 \\
(0.000)\end{array}$ & 0.033 \\
\hline & $P_{20}\left(\mathrm{~mm} / \mathrm{rad}^{2}\right)$ & $\begin{array}{c}225.96 \\
(226.65)\end{array}$ & 216.71 & $\begin{array}{c}202.68 \\
(203.20)\end{array}$ & -5.4729 & $\begin{array}{c}190.14 \\
(190.59)\end{array}$ & 130.01 \\
\hline$t_{\pi}$ & $P_{10}^{\prime}(\mathrm{ns} / \mathrm{rad})$ & $\begin{array}{c}39.848 \\
(39.848)\end{array}$ & 38.355 & $\begin{array}{c}43.338 \\
(43.337)\end{array}$ & 43.927 & $\begin{array}{l}57.542 \\
(57.541)\end{array}$ & 45.546 \\
\hline & $P_{30}^{\prime}\left(\mathrm{ns} / \mathrm{rad}^{2}\right)$ & $\begin{array}{r}-39.4739 \\
(-39.34)\end{array}$ & -30.260 & $\begin{array}{c}-36.0783 \\
(-36.11)\end{array}$ & -38.169 & $\begin{array}{c}-42.8798 \\
(-43.19)\end{array}$ & -38.532 \\
\hline
\end{tabular}

are rather low $(\leq \mathrm{pA})$ as count rates are typically less than $100 \mathrm{kHz}$. In particular, in coincidence experiments, where the transit times are of particular interest, count rates are even smaller in an effort to maintain an acceptable true/ random coincidence ratio. Therefore, in both these applications space charge effects can be expected to be negligible and for this reason we have not considered them in our presentation.

Finally, we note some points relating to the accuracy of the simulations. As the HDA system has curved electrodes that do not perfectly align to square grid points, we have used the "surface enhancement" feature of SIMION 8.1 to improve the accuracy of the surface regions modeled [36]. The improvement in the field accuracy is expected to be at least an order of magnitude over that reported in [50]. In addition, since relativistic effects are not considered in the classical theory of motion presented, we have used nonrelativistic trajectories (an option provided by SIMION in its otherwise fully relativistic treatment of the motion) in the ideal field calculations shown in the contour plots of Figures 4-7 and the results extracted from Figure 7 and shown in Table 4.

\section{Results and Discussion}

The comparison of the three entry positions, $R_{0}=82.55 \mathrm{~mm}$, $101.6 \mathrm{~mm}$, and $116 \mathrm{~mm}$, involved calculations of electron trajectories having otherwise identical analyzer parameters. From these simulations, a detailed comparison of the electrons' spatial ( $x$ and $y$ ) distributions and kinetic energy variations with time was made. In Table 2 we present a summary of the electron optical properties. As can be seen from Table 2, in the fringing field HDAs the 1st-order focusing is achieved (aberration coefficient $P_{10} \approx 0$ ) in both paracentric cases.
Note that 2nd-order focusing is not possible in any type of HDA (i.e., $P_{20} \neq 0$ ) but only in the full spherical electrostatic capacitor for complete orbits [54].

Figure 3 shows the time-of-flight $t_{\pi}$ and the exit radius $r_{\pi}$ as a function of the maximum pencil angle for the three different entry positions. As also shown in Table 3, the time-of-flight values for the central trajectory $(\alpha=0)$ are $14.300 \mathrm{~ns}\left(R_{0}=82.55 \mathrm{~mm}\right), 19.892 \mathrm{~ns}\left(R_{0}=101.6 \mathrm{~mm}\right)$, and $21.880 \mathrm{~ns}\left(R_{0}=116 \mathrm{~mm}\right)$, respectively, at a pass energy of $E_{0}=1 \mathrm{keV}$. The time-of-flight values along the central $(\alpha=0)$ trajectories given by (28) in the ideal HDA are shorter than that in the fringing field HDA. For $R_{0}=116 \mathrm{~mm}$ with the entry bias $\gamma=0.499$, the time peaks shift to longer times. In this case, the time-of-flight increases, since the electrons are slowed down in the analyzer for $\gamma<1.0$ and because their paths $s_{\pi}$ are longer than for the other entry positions. For $R_{0}=82.55 \mathrm{~mm}$ with $\gamma \approx 1.5$ the electron velocity increases since they are now accelerated by the entry bias, shortening the electron drift time across the region with electrostatic field.

The exit position for entry $R_{0}=101.6 \mathrm{~mm}$ in Figure 3(d) is seen to be asymmetric with respect to the central trajectory $(\alpha=0)$, greatly increasing its spread in position across the detector plane. For the paracentric cases (with the optimized values of $R_{0}$ and $\gamma$ ) the electron trajectories show near ideal performance and the exit positions are seen to be symmetric in the exit plane. In this case, the exit positions are nearly ideal compared with the same traces in a truly ideal field in Figure 3(b).

In Figures 4-7 we show our HDA results in the form of contour plots for each of the three entry radii $R_{0}$. The calculated exit spot sizes are shown on the detector plane ( $x y$-plane) as time and energy distributions. The changes in the time-of-flight depend on the entry beam size $\Gamma$, 
TABLE 3: This is as in Table 2 but for the time-of-flight $t_{\pi}$ and the length of the flown trajectory $s_{\pi}$ of electrons starting with different launching angles $\alpha$.

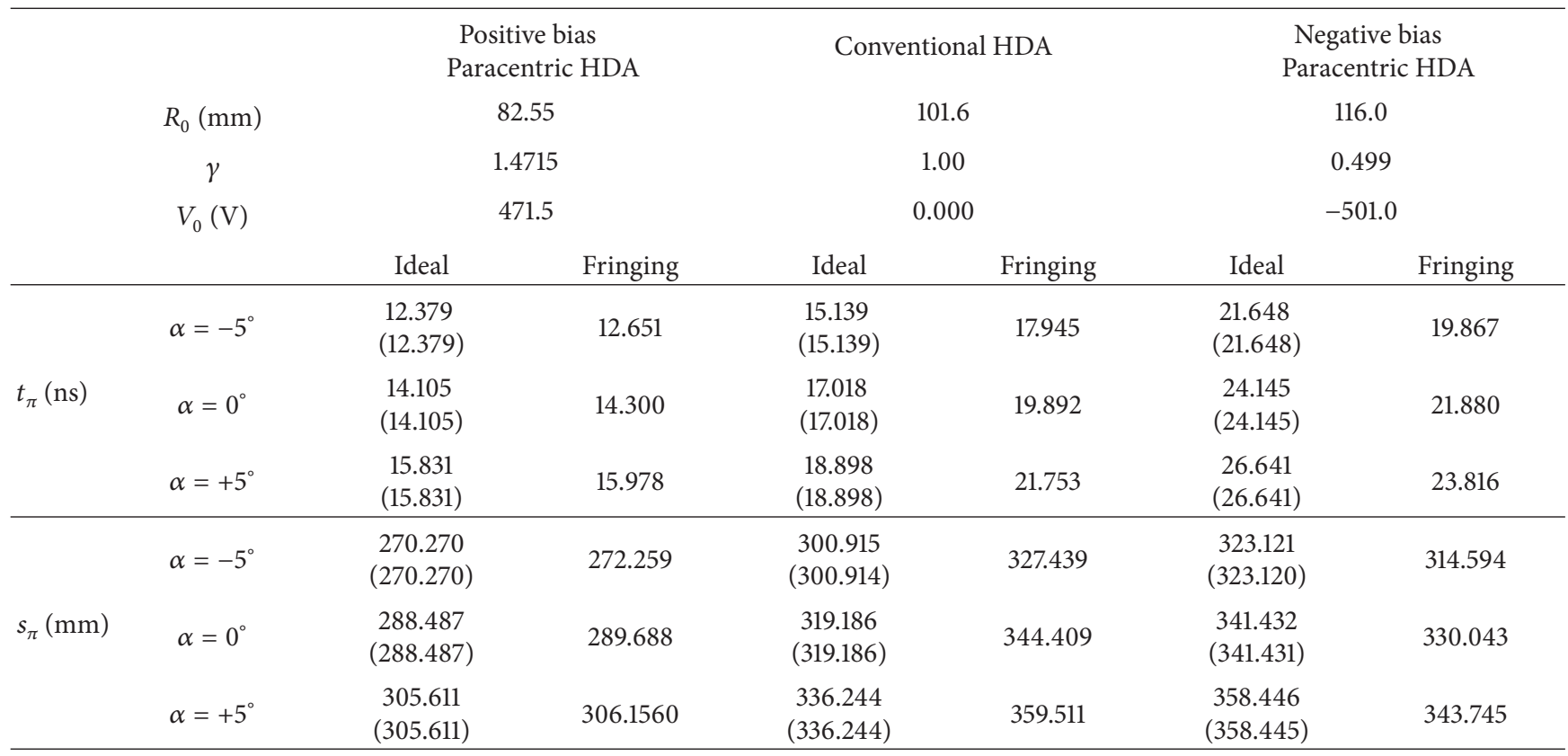

the source energy spread $\delta E$, and the limiting launching angle $\alpha_{\max }$. The calculated time-of-flight line shapes for a parallel monoenergetic input beam $\left(\delta E=0\right.$ and $\left.\alpha_{\max }=0\right)$ are shown in Figure 4 for source extent $\Gamma=0.5,1.0$, and $1.5 \mathrm{~mm}$. The time spread of the electrons was found to increase linearly as $\Gamma$ increases. For $R_{0}=101.6 \mathrm{~mm}$, the time-of-flight deviations due to the finite source extent are seen not to contribute significantly to the time spread, because the differences in electron optical paths are negligible. The narrow base width in the $t_{\pi}$ distribution for $R_{0}=101.6 \mathrm{~mm}$ can be understood by inspection of the nominal entry bias potential $V\left(R_{0}\right)$, which is zero, and therefore electrons travel along or very near the zero-equipotential line. For the two paracentric entries, however, for which $V\left(R_{0}\right) \neq 0$, the effect of $\Gamma$ on $t_{\pi}$ cannot be ignored.

We have also investigated the contributions of the launching angle to the time spread, calculating the flight time through the HDA for an electron of energy $E_{0}$ with varying launching angle between $-\alpha_{\max }$ and $+\alpha_{\max }$. The time profile of electrons for HDAs for the three different values of $R_{0}$ and a range of pencil angles are shown in Figure 5. Clearly, the width of the time-of-flight distribution varies significantly with the entrance angle, even in the case of a monoenergetic electron beam. With the angles varying from $1^{\circ}$ to $5^{\circ}$, the aberrations degrade the time resolution of the analyzer in all three cases. The simulations show that even though the focusing becomes poorer for $R_{0}=101.6 \mathrm{~mm}$, the time spread is not much affected by the fringing fields. In addition, one can see from Figure 5 that the time spread increases rapidly with increasing pencil angle, but the full width at half maximum $\left(\Delta t_{\pi}(50 \%)\right)$ is not increased as much as the base width in either case, which can lead to significant misconceptions about the true time spread for the HDAs.
Another limitation of the temporal resolution of the HDA is given by the energy spread of the source. In Figure 6, the same calculations have been repeated as those shown in Figures 4 and 5 where the electrons were monoenergetic. However, now the energy of the electrons has been smeared out around the pass energy by $\delta E=2 \mathrm{eV}, 3 \mathrm{eV}$, and $4 \mathrm{eV}$ but assuming no angular deviations. The electrons with a given initial energy spread $\delta E$ (FWHM) were generated using a Gaussian distribution. Higher energy electrons penetrate more deeply into the field of the HDA, leading to a longer path length and different arrival times at the exit of the analyzer. It is clear that (i) the time spread increases strongly with $\delta E$ and (ii) the biased paracentric HDA with $R_{0}=116 \mathrm{~mm}$ has larger time spreads compared to the other two entries due to its higher energy dispersion $D_{\gamma}$ [33]. Overall, a small deviation in the energy spread is seen to cause but a slight increase in the time spread for all cases.

In Figure 7, an electron source with both energy and angular distribution is considered, and four data sets are taken with finite source extent: $\Gamma=0.5 \mathrm{~mm}, \delta E=2 \mathrm{eV}$, and $\alpha_{\max }=3^{\circ}$. The ideal field calculation for $R_{0}=101.6 \mathrm{~mm}$ is given in Figure 7(a) for reference. The simulation parameters and calculated resolutions are also given in Table 4 . Since the line shapes in Figure 7 are mostly Gaussian, base widths are not considered, but the widths at $1 \%\left(\Delta t_{\pi}(1 \%)\right)$ and at $50 \%\left(\Delta t_{\pi}(50 \%)\right)$ of the peak height are presented. This comparison shows that an improvement in time and energy spreads is obtained for the biased paracentric case with $R_{0}=$ $82.55 \mathrm{~mm}$ compared to the conventional centric HDA. The time and energy FWHM for the HDA with $R_{0}=82.55 \mathrm{~mm}$ are $\Delta t_{\pi}(50 \%)=1.70 \mathrm{~ns}$ and $\Delta E(50 \%)=4.07 \mathrm{eV}$, respectively, while those for $R_{0}=116 \mathrm{~mm}$ are $1.98 \mathrm{~ns}$ and $3.17 \mathrm{eV}$ and for $R_{0}=101.6 \mathrm{~mm}$ they are $1.98 \mathrm{~ns}$ and $20.3 \mathrm{eV}$, respectively. 


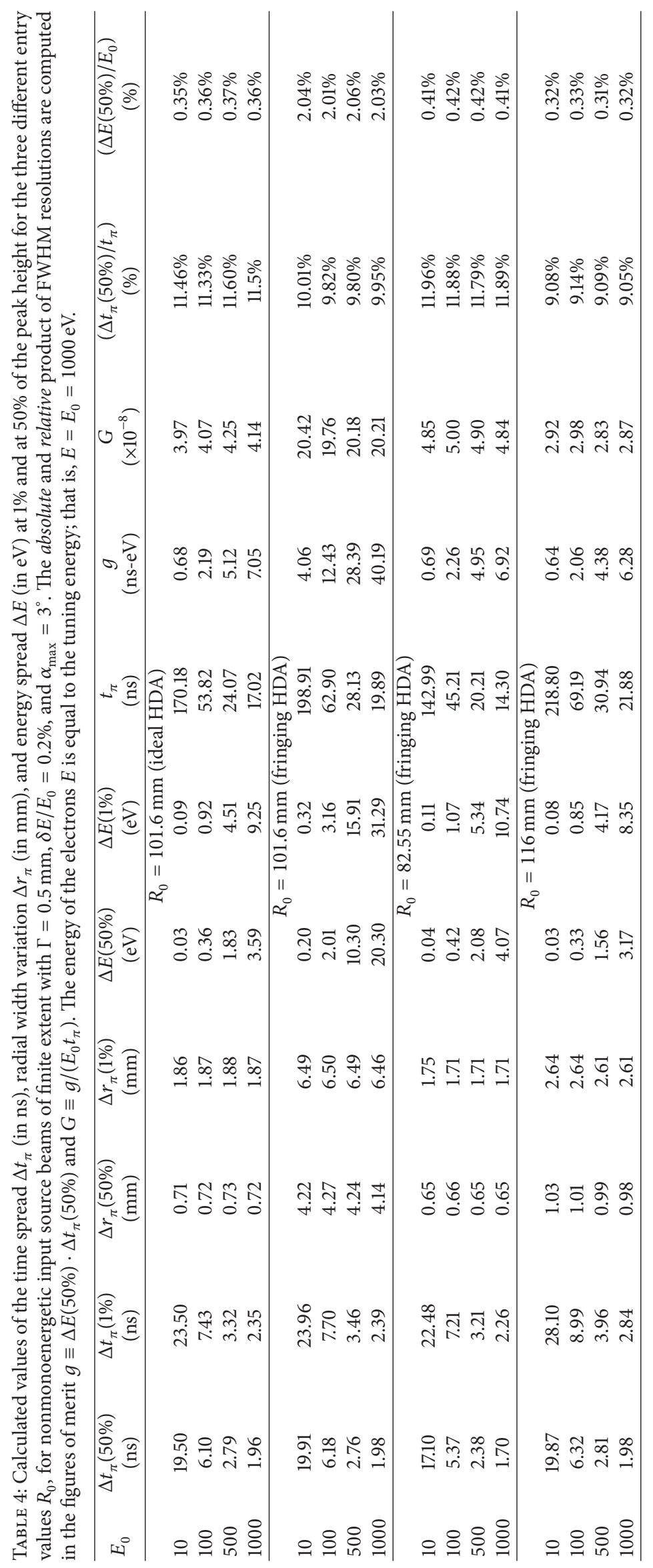



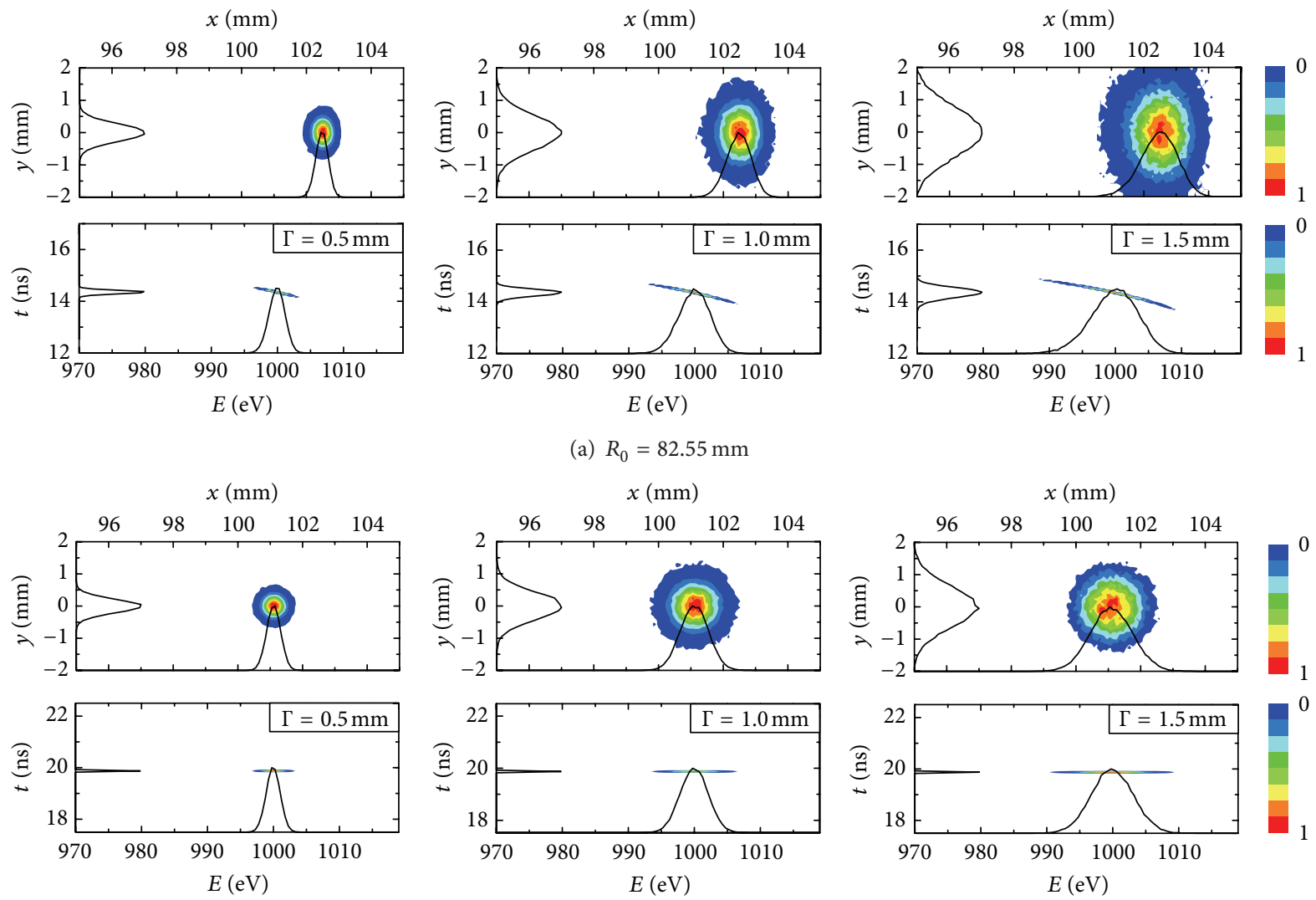

(a) $R_{0}=82.55 \mathrm{~mm}$
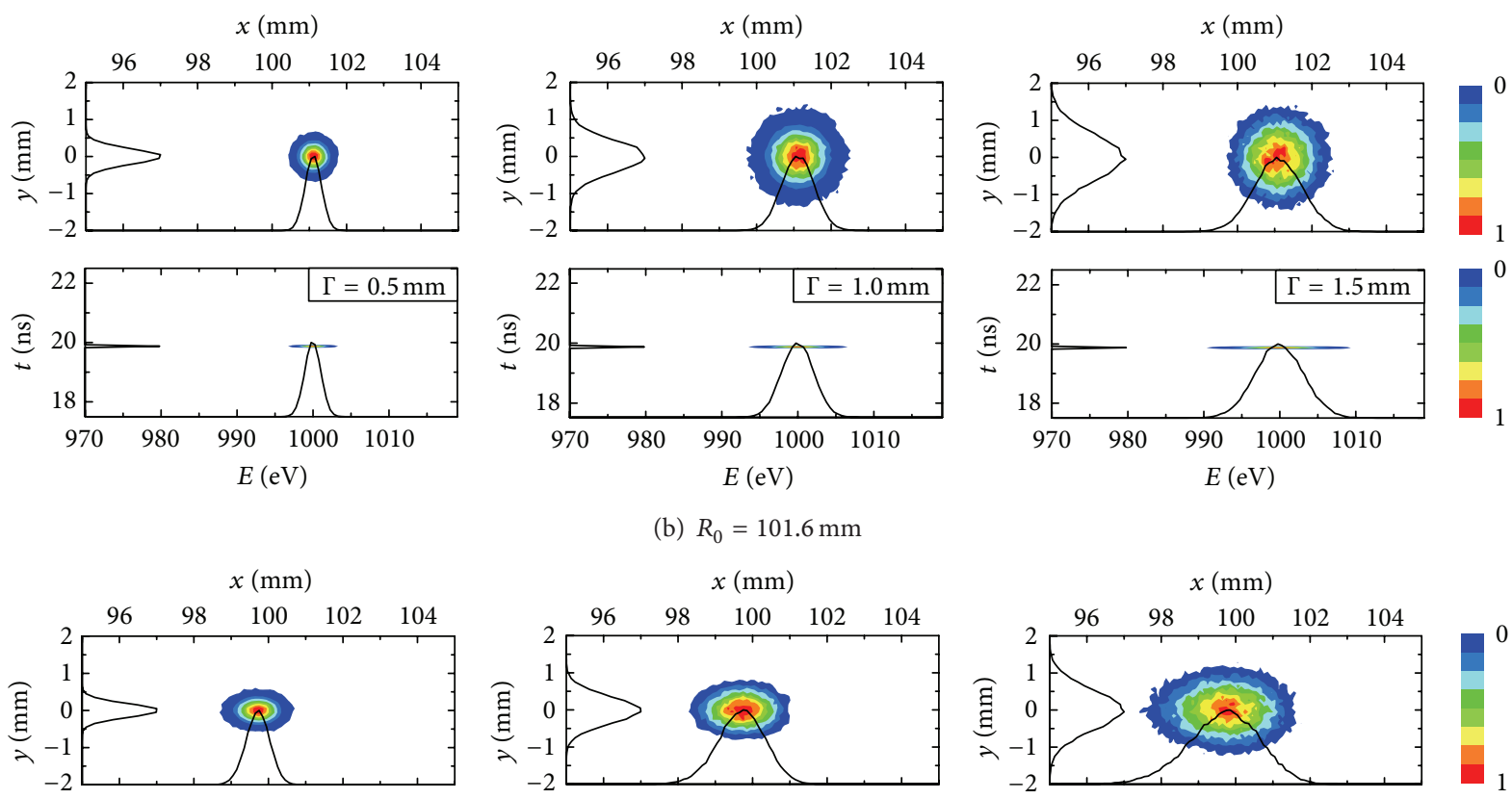

(b) $R_{0}=101.6 \mathrm{~mm}$
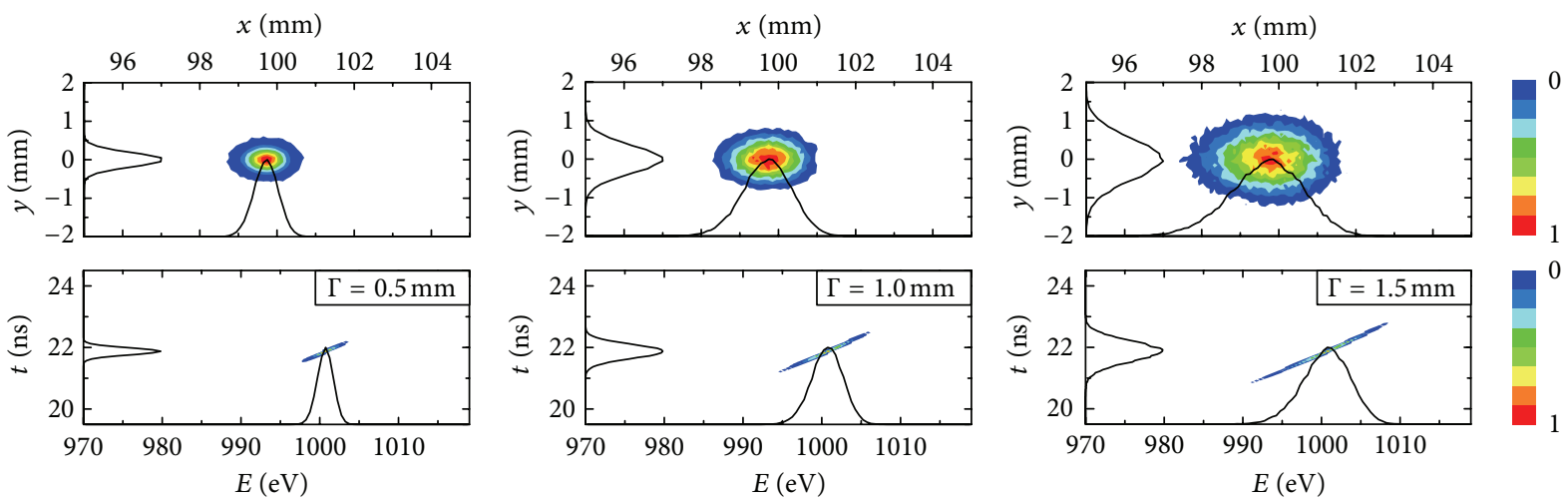

(c) $R_{0}=116 \mathrm{~mm}$

FIGURE 4: Calculated spot size on the detector plane ( $x y$-plane) and corresponding time-energy distributions at the exit of the HDA for three different values of the HDA entry radius $R_{0}:$ (a) $82.55 \mathrm{~mm}$, (b) $101.6 \mathrm{~mm}$, and (c) $116 \mathrm{~mm}$, and for a monoenergetic parallel beam: $\delta E=0 \mathrm{eV}$, $\alpha_{\max }=0$, and $E_{0}=1 \mathrm{keV}$. Simulations were performed for source extent: $\Gamma=0.5,1.0$, and $1.5 \mathrm{~mm}$. Normalized line profiles resulting from vertical and horizontal projections of the electron distributions are also shown. The detector plane is assumed to coincide with the HDA exit plane.

In Table 4, the variation of the time and energy spreads is tabulated for different pass energies $E_{0}$, assuming that both the initial angular and the spatial distributions are kept constant. The time spread is seen to be inversely proportional to the square root of the pass energy, that is, $\Delta t \propto E_{0}^{-1 / 2}$. This means that the reduction of the time-of-flight spread can be achieved by increasing the pass energy. However, an increase of the pass energy leads to a decrease in the energy resolution. Therefore, a suitable compromise between good energy resolution (favoring smaller $E_{0}$ ) and good time resolution (favoring larger $E_{0}$ ) must be found. With this in mind, we have introduced two figures of merits, $g \equiv$ $\Delta E(50 \%) \cdot \Delta t_{\pi}(50 \%)$ and $G \equiv g /\left(E_{0} t_{\pi}\right)$, for the detailed intercomparison of the joint time and energy resolution properties of the HDAs. In Figure 8, both $g$ and $G$ are plotted for four values of $E_{0}$. For each $R_{0}$, we find an almost constant 

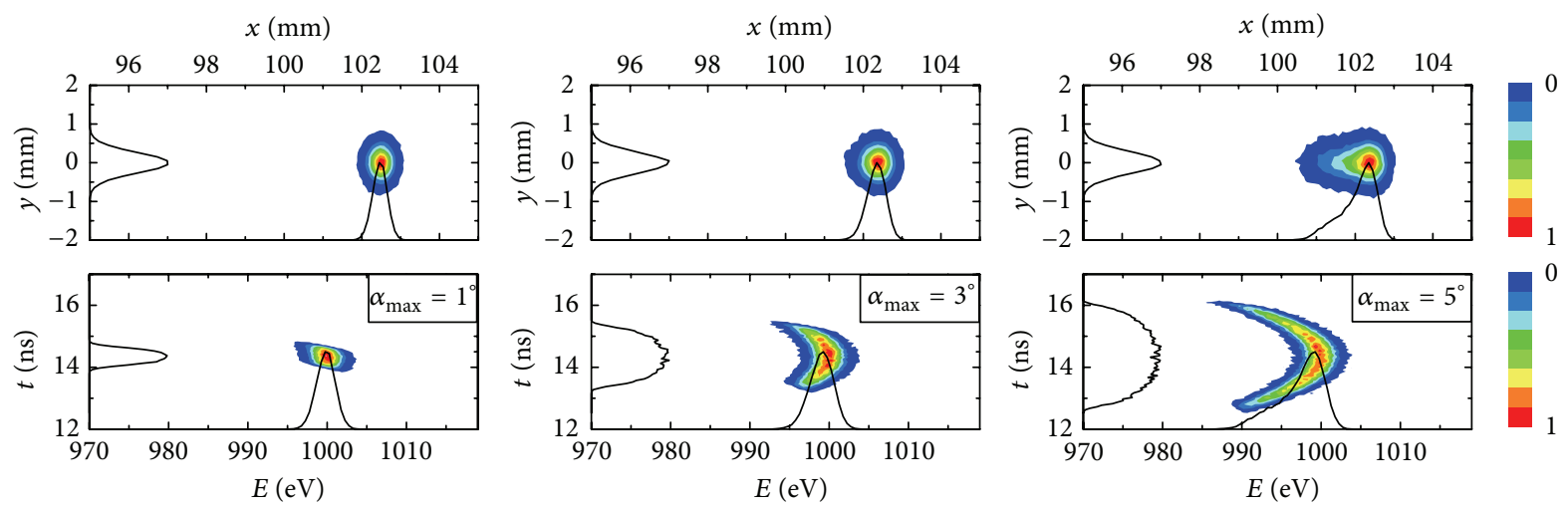

(a) $R_{0}=82.55 \mathrm{~mm}$
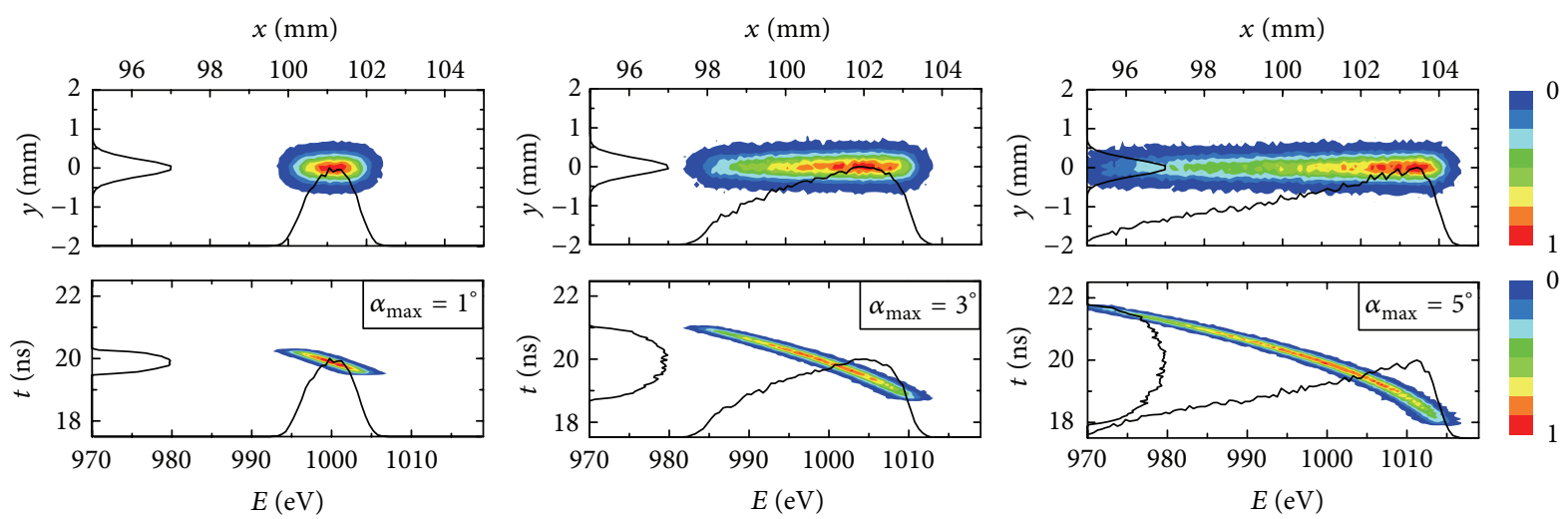

(b) $R_{0}=101.6 \mathrm{~mm}$
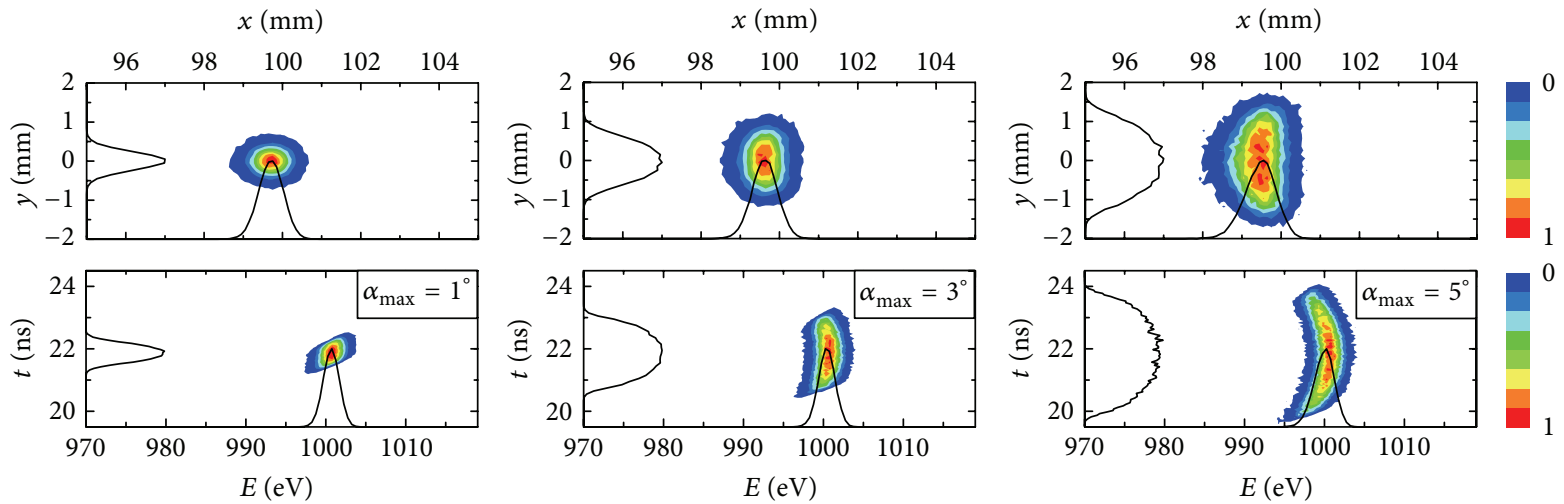

(c) $R_{0}=116 \mathrm{~mm}$

Figure 5: This is the same as Figure 4 but for electrons having uniform and random launching angles $\alpha \leq\left|\alpha_{\max }\right|$ with $\alpha_{\max }=1^{\circ}, 3^{\circ}$, and $5^{\circ}$ and $\delta E=0 \mathrm{eV}, \Gamma=0.5 \mathrm{~mm}$, and $E_{0}=1 \mathrm{keV}$.

$G$ because the relative resolutions are constant. In the case of fringing field HDAs, the two paracentric entries give a smaller value of $g$ and $G$ than the conventional centric entry.

\section{Summary and Conclusions}

The biased paracentric HDA differs from the conventional HDA in two important ways: (a) the entry distance $R_{0}$ is paracentric, that is, either larger or smaller than the mean HDA radius used in a conventional HDA, and (b) the two hemispherical electrode voltages are set so that the HDA entry bias potential $V\left(R_{0}\right)$ is nonzero (biased), compared to conventional HDA usage in which $V\left(R_{0}\right)=$ 0 . For very particular value combinations of $R_{0}$ and $V\left(R_{0}\right)$, empirically found through simulations, we have shown in the past that substantial improvements in energy resolution can be attained, with 1st-order focusing conditions practically restored without the use of any additional fringing field 

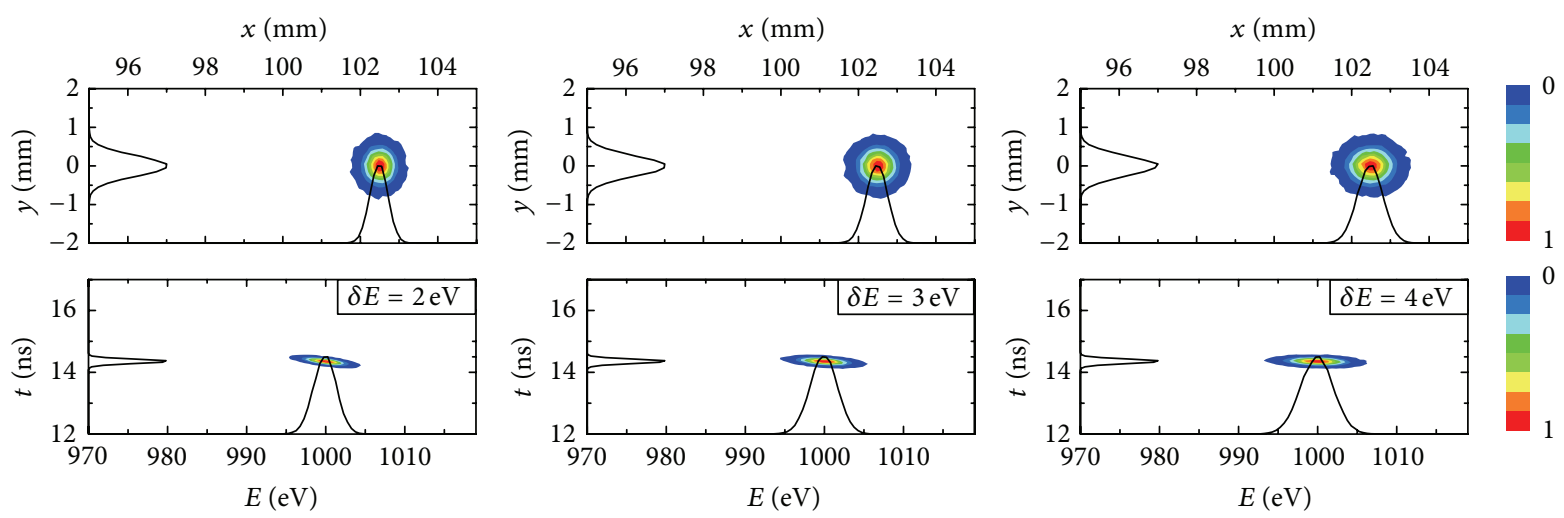

(a) $R_{0}=82.55 \mathrm{~mm}$
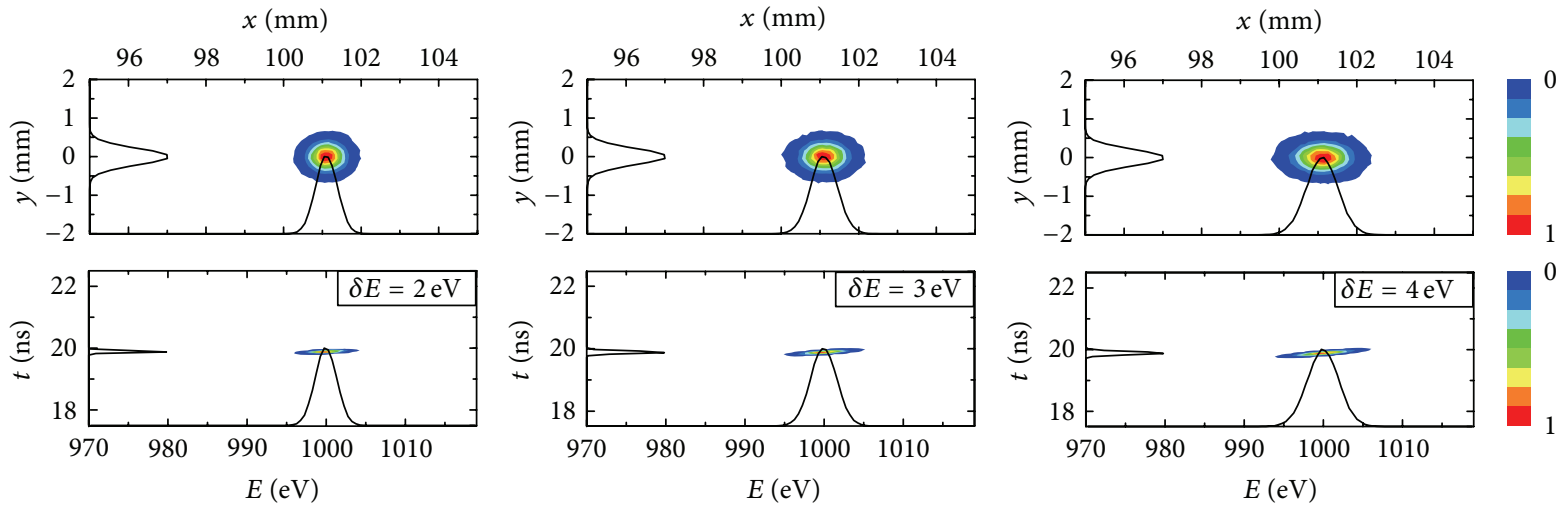

(b) $R_{0}=101.6 \mathrm{~mm}$
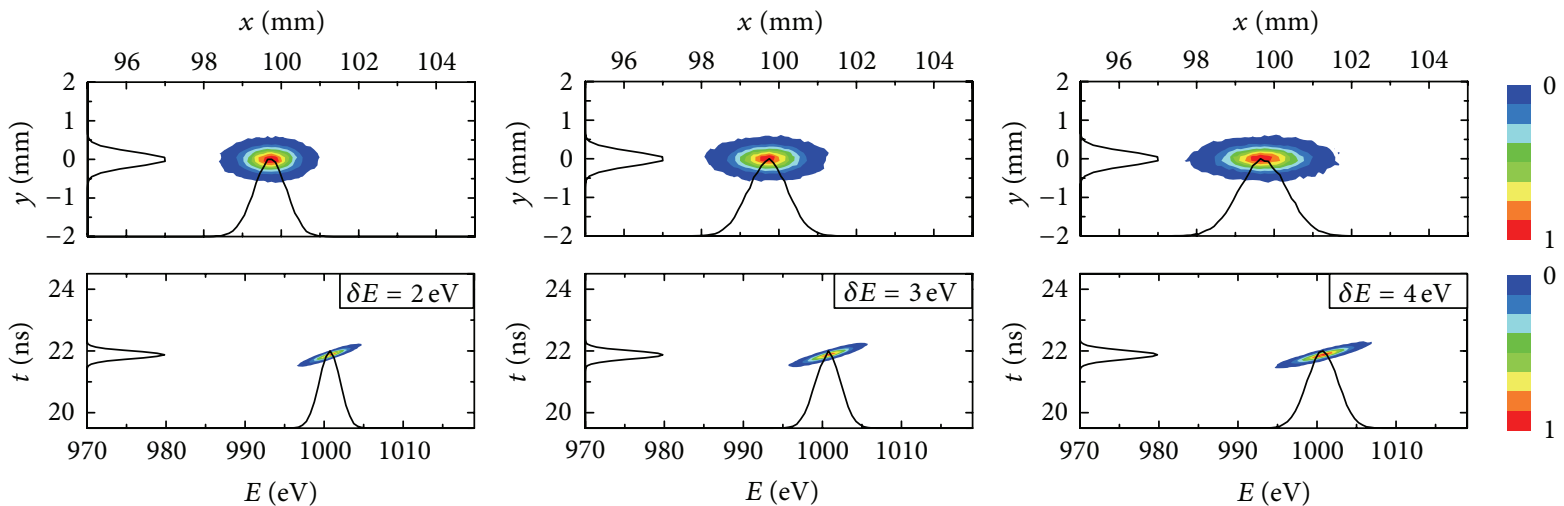

(c) $R_{0}=116 \mathrm{~mm}$

FIGURE 6: This is the same as Figure 4 but for nonmonoenergetic beams with given source energy spread (FWHM) $\delta E=2,3$, and $4 \mathrm{eV}$ for source extent $\Gamma=0.5 \mathrm{~mm}, \alpha_{\max }=0$, and $E_{0}=1 \mathrm{keV}$.

correction electrodes, as typically done for conventional HDAs.

Here, we have explored for the first time the time-offlight properties of the biased paracentric HDA for the two special entry positions already investigated in the past as to their energy resolution and compared them to those of the conventional HDA. The trajectory related time spreads for electrons were calculated numerically since their flight times are not predictable analytically in the fringing field HDA. Our analysis quantified the effect of varying the controllable input variables of entry position, source extent, launching angle, and energy spread on selected output variables of time-offlight and time spread.

When one considers a realistic distribution with energy spread, angular spread, and finite source extent, both biased paracentric entries were found to have near ideal field 

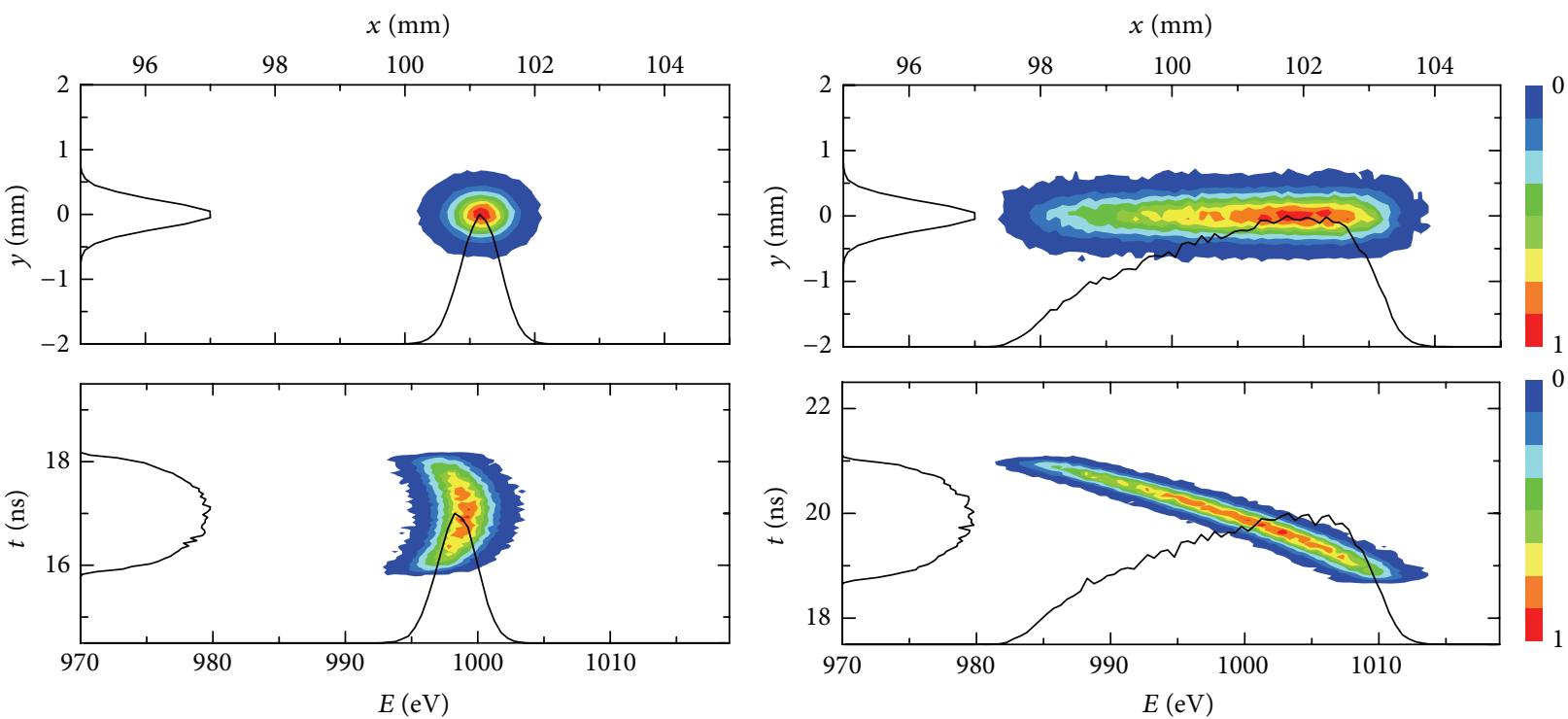

(a) Ideal HDA

(b) $R_{0}=101.6 \mathrm{~mm}$
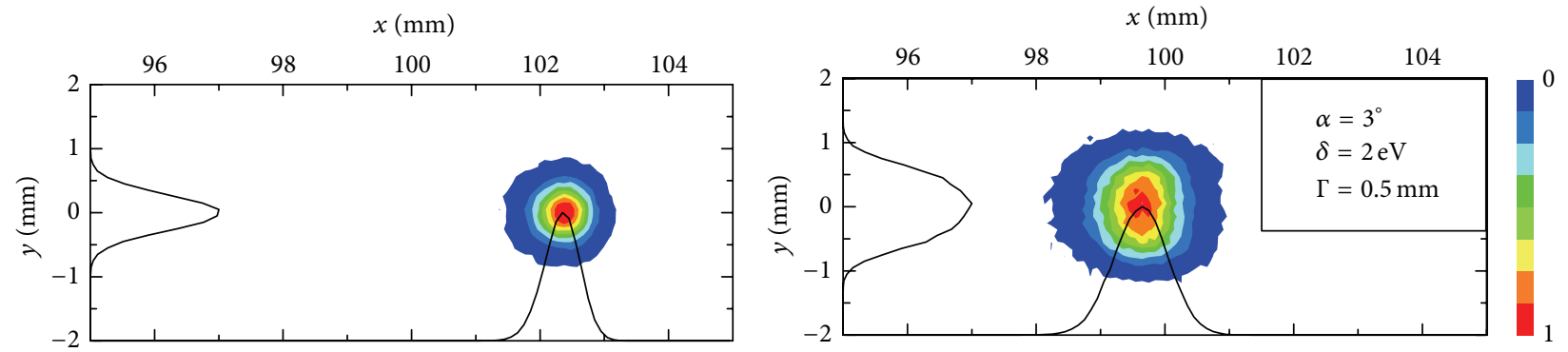

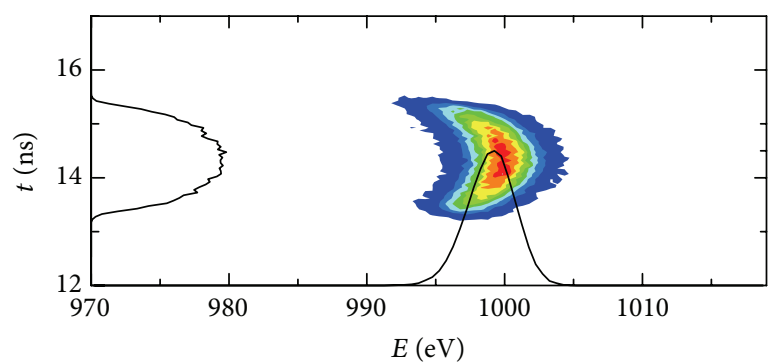

(c) $R_{0}=82.55 \mathrm{~mm}$

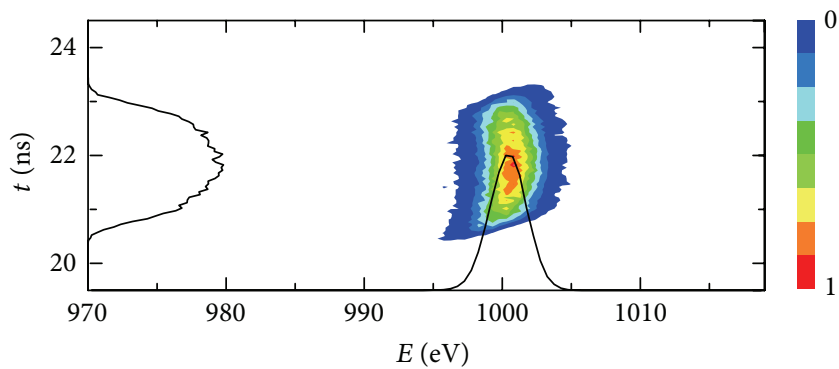

(d) $R_{0}=116 \mathrm{~mm}$

FIgURE 7: This is the same as Figure 6 but with finite extent: $\Gamma=0.5 \mathrm{~mm}, \delta E=2 \mathrm{eV}, \alpha_{\max }=3^{\circ}$, and $E_{0}=1 \mathrm{keV}$. In addition, the ideal field calculation is also shown in (a) for $R_{0}=101.6 \mathrm{~mm}$. The two paracentric entries for the fringing field HDA are seen in (c) and (d) to have near ideal field HDA distributions.

behavior with the $R_{0}=82.55 \mathrm{~mm}$ entry showing better absolute energy and time distributions and the $R_{0}=116 \mathrm{~mm}$ entry, showing better relative energy and time distributions and their products, the figures of merit $g$ and $G$ (see Table 4). Thus, overall, both biased paracentric entries should prove themselves to be superior to the conventional HDA entry both in timing and in energy resolution applications offering an attractive alternative for combined timing and energy resolution applications such as coincidence experiments, where electrons detected in the HDA with high resolution must also be related to events in other detectors for the same event. It is our hope that these results will stimulate further interest in the construction and testing of such HDAs.

\section{Conflict of Interests}

The authors declare that there is no conflict of interests regarding the publication of this paper. 


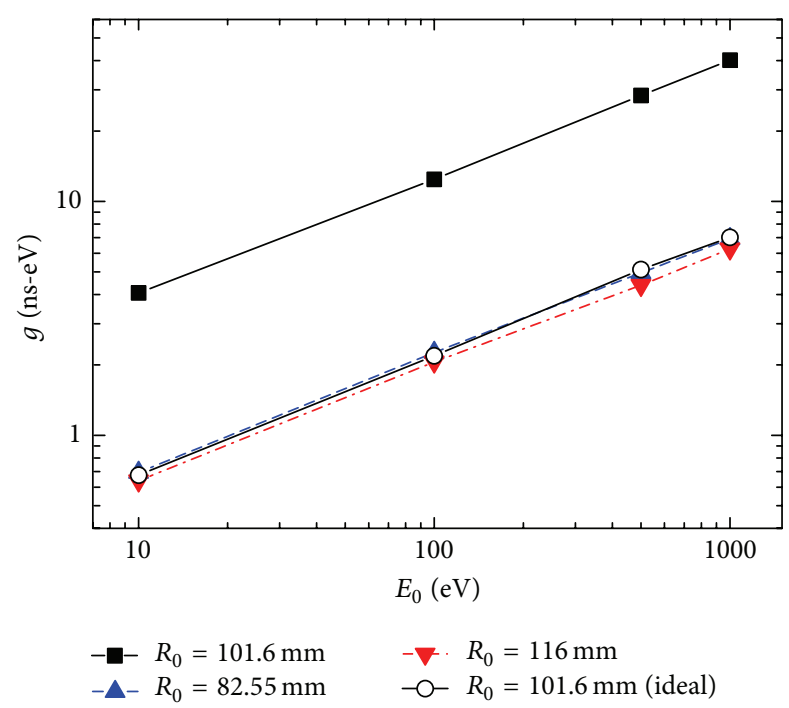

(a)

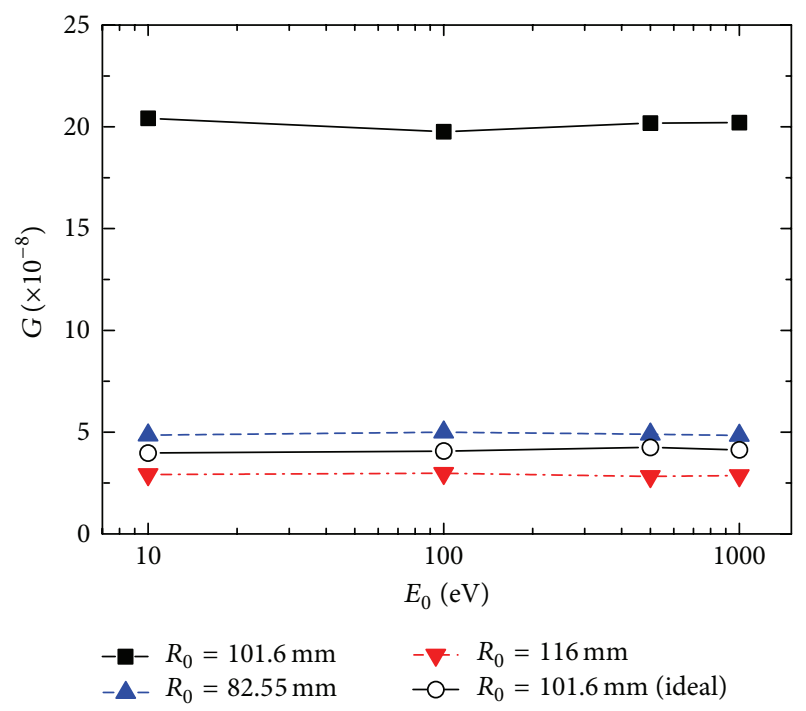

(b)

FIGURE 8: Variation of the figures of merit $g \equiv \Delta E(50 \%) \cdot \Delta t_{\pi}(50 \%)$ and $G \equiv g /\left(E_{0} t_{\pi}\right)$ with pass energy $E_{0}$. In addition, the ideal field results are also shown for $R_{0}=101.6 \mathrm{~mm}$. The two fringing field paracentric entries are seen to have near ideal field behavior with the entry at $R_{0}=116 \mathrm{~mm}$ showing a slight superiority in both figures of merit.

\section{Acknowledgments}

This research has been cofinanced by the European Union (European Social Fund, ESF) and Greek national funds through the Operational Program "Education and Lifelong Learning" of the National Strategic Reference Framework (NSRF) Research Funding Program: THALES. Investing in knowledge society was through the European Social Fund, Grant number MIS 377289. The authors would like to thank David J. Manura of SIS for his help with various SIMION issues.

\section{References}

[1] T. J. M. Zouros and E. P. Benis, "The hemispherical deflector analyser revisited. I. Motion in the ideal $1 / r$ potential, generalized entry conditions, Kepler orbits and spectrometer basic equation," Journal of Electron Spectroscopy and Related Phenomena, vol. 125, no. 3, pp. 221-248, 2005, Erratum in: Journal of Electron Spectroscopy and Related Phenomena, vol. 142, pp. 175-176, 2005.

[2] D. Roy and D. Tremblay, "Design of electron spectrometers," Reports on Progress in Physics, vol. 53, no. 12, pp. 1621-1674, 1990.

[3] B. Wannberg, "Electron optics development for photo-electron spectrometers," Nuclear Instruments and Methods in Physics Research, Section A: Accelerators, Spectrometers, Detectors and Associated Equipment, vol. 601, no. 1-2, pp. 182-194, 2009.

[4] Y. Ballu, "High resolution electron spectroscopy," in Advances in Electronics and Electron Physics, pp. 1192-1101, Academic Press, 1980.

[5] E. Granneman and M. van der Wiel, "Transport, dispersion and detection of electrons, ions and neutrals," in Handbook of Synchrotron Radiation, vol. 1, 1983.
[6] N. Mårtensson, P. Baltzer, P. A. Brühwiler et al., "A very high resolution electron spectrometer," Journal of Electron Spectroscopy and Related Phenomena, vol. 70, no. 2, pp. 117-128, 1994.

[7] E. P. Benis and T. J. M. Zouros, "The hemispherical deflector analyser revisited: II. Electron-optical properties," Journal of Electron Spectroscopy and Related Phenomena, vol. 163, no. 1-3, pp. 28-39, 2008.

[8] A. Shavorskiy, S. Neppl, D. S. Slaughter et al., "Sub-nanosecond time-resolved ambient-pressure X-ray photoelectron spectroscopy setup for pulsed and constant wave X-ray light sources," Review of Scientific Instruments, vol. 85, no. 9, Article ID 093102, 2014.

[9] O. Sise, M. Dogan, I. Okur, and A. Crowe, "Electron-impact excitation of the $\left(2 p^{2}\right){ }^{1} D$ and $(2 s 2 p){ }^{1} P^{o}$ autoionizing states of helium," Physical Review A, vol. 84, no. 2, Article ID 022705, 2011.

[10] M. Volkel and W. Sandner, "Optimisation of electron energy analysers for application in coincidence experiments," Journal of Physics E: Scientific Instruments, vol. 16, no. 5, pp. 456-462, 1983.

[11] R. W. Schoenlein, S. Chattopadhyay, H. H. W. Chong et al., "Generation of femtosecond pulses of synchrotron radiation," Science, vol. 287, no. 5461, pp. 2237-2240, 2000.

[12] W. Ackermann, G. Asova, V. Ayvazyan et al., "Operation of a free-electron laser from the extreme ultraviolet to the water window," Nature Photonics, vol. 1, no. 6, pp. 336-342, 2007.

[13] S. Hellmann, C. Sohrt, M. Beye et al., "Time-resolved X-ray photoelectron spectroscopy at FLASH," New Journal of Physics, vol. 14, no. 1, Article ID 013062, 2012.

[14] T. Ishikawa, H. Aoyagi, T. Asaka et al., "A compact X-ray freeelectron laser emitting in the sub-angstrom region," Nature Photonics, vol. 6, no. 8, pp. 540-544, 2012.

[15] P. Emma, R. Akre, J. Arthur et al., "First lasing and operation of an ångstrom-wavelength free-electron laser," Nature Photonics, vol. 4, no. 9, pp. 641-647, 2010. 
[16] N. Berrah, L. Fang, B. Murphy et al., "Double-core-hole spectroscopy for chemical analysis with an intense X-ray femtosecond laser," Proceedings of the National Academy of Sciences of the United States of America, vol. 108, no. 41, pp. 16912-16915, 2011.

[17] K. Appel, M. Nakatsutsumi, A. Pelka, G. Priebe, I. Thorpe, and T. Tschentscher, "Studying planetary matter using intense $\mathrm{x}-$ ray pulses," Plasma Physics and Controlled Fusion, vol. 57, no. 1, Article ID 014003, 2015.

[18] A. Shavorskiy, A. Cordones, J. Vura-Weis et al., “Time-resolved $\mathrm{X}$-ray photoelectron spectroscopy techniques for real-time studies of interfacial charge transfer dynamics," AIP Conference Proceedings, vol. 1525, no. 1, pp. 475-479, 2013.

[19] S. Neppl and O. Gessner, “Time-resolved X-ray photoelectron spectroscopy techniques for the study of interfacial charge dynamics," Journal of Electron Spectroscopy and Related Phenomena, 2015.

[20] L. Nugent-Glandorf, M. Scheer, D. A. Samuels et al., "Ultrafast time-resolved soft X-ray photoelectron spectroscopy of dissociating $\mathrm{Br}_{2}$," Physical Review Letters, vol. 87, no. 19, Article ID 193002, 2001.

[21] P. Siffalovic, M. Drescher, and U. Heinzmann, "Femtosecond time-resolved core-level photoelectron spectroscopy tracking surface photovoltage transients on p-GaAs," Europhysics Letters, vol. 60, no. 6, pp. 924-930, 2002.

[22] J. Chen and P. M. Rentzepis, "Subpicosecond and sub-angstrom time and space studies by means of light, X-ray, and electron interaction with matter," Journal of Physical Chemistry Letters, vol. 5, no. 1, pp. 225-232, 2014.

[23] T. Popmintchev, M.-C. Chen, D. Popmintchev et al., "Bright coherent ultrahigh harmonics in the $\mathrm{keV} \mathrm{X}$-ray regime from mid-infrared femtosecond lasers," American Association for the Advancement of Science. Science, vol. 336, no. 6086, pp. 12871291, 2012.

[24] R. E. Imhof, A. Adams, and G. C. King, "Energy and time resolution of the 180 degrees hemispherical electrostatic analyser," Journal of Physics E: Scientific Instruments, vol. 9, no. 2, p. 5, 1976.

[25] R. S. Caprari, "Exit position and transit-time analysis of hemispherical electrostatic analysers," Measurement Science \& Technology, vol. 6, no. 7, pp. 1063-1064, 1995.

[26] O. Kugeler, S. Marburger, and U. Hergenhahn, "Calculation and measurement of the time-of-flight spread in a hemispherical electron energy analyzer," Review of Scientific Instruments, vol. 74, no. 9, pp. 3955-3961, 2003.

[27] E. P. Benis and T. J. M. Zouros, "Improving the energy resolution of a hemispherical spectrograph using a paracentric entry at a non-zero potential," Nuclear Instruments and Methods in Physics Research, Section A: Accelerators, Spectrometers, Detectors and Associated Equipment, vol. 440, no. 2, pp. 462-465, 2000.

[28] E. P. Benis, K. Zaharakis, M. M. Voultsidou et al., "A new hemispherical analyser with 2-D PSD and focusing lens for use in 0。 electron spectroscopy," Nuclear Instruments and Methods in Physics Research Section B: Beam Interactions with Materials and Atoms, vol. 146, no. 1-4, pp. 120-125, 1998.

[29] M. Dogan, M. Ulu, G. G. Gennarakis, and T. J. M. Zouros, "Experimental energy resolution of a paracentric hemispherical deflector analyzer for different entry positions and bias," Review of Scientific Instruments, vol. 84, no. 4, Article ID 043105, 2013.

[30] The APAPES Collaboration, http://apapes.physics.uoc.gr/.
[31] S. Doukas, I. Madesis, A. Dimitriou, A. Laoutaris, T. J. Zouros, and E. P. Benis, "Determination of the solid angle and response function of a hemispherical spectrograph with injection lens for Auger electrons emitted from long lived projectile states," Review of Scientific Instruments, vol. 86, no. 4, Article ID 043111, 2015.

[32] O. Sise, "Simulation of electron energy spectra of a biased paracentric hemispherical deflection analyzer as a function of entry bias: effects of misalignments," Journal of Spectroscopy, vol. 2014, Article ID 152647, 8 pages, 2014.

[33] O. Sise, M. Ulu, M. Dogan, G. Martinez, and T. J. M. Zouros, "Fringing field optimization of hemispherical deflector analyzers using BEM and FDM," Journal of Electron Spectroscopy and Related Phenomena, vol. 177, no. 1, pp. 42-51, 2010.

[34] O. Sise, T. J. M. Zouros, M. Ulu, and M. Dogan, "Novel and traditional fringing field correction schemes for the hemispherical analyser: comparison of first-order focusing and energy resolution," Measurement Science \& Technology, vol. 18, no. 7, pp. 1853-1858, 2007.

[35] T. J. M. Zouros, O. Sise, M. Ulu, and M. Dogan, "Using the fringing fields of a hemispherical spectrograph to improve its energy resolution," Measurement Science \& Technology, vol. 17, no. 12, pp. N81-N86, 2006.

[36] SIMION Version 8.1.1.29, Scientific Instrument Services, Ringoes, NJ, USA, http://www.simion.com.

[37] E. M. Purcell, "The focusing of charged particles by a spherical condenser," Physical Review, vol. 54, no. 10, pp. 818-826, 1938.

[38] M. Yavor, "Electrostatic energy analyzers," Optics of Charged Particle Analyzers, vol. 157, pp. 213-258, 2009.

[39] A. Khursheed, Scanning Electron Microscope Optics and Spectrometers, World Scientific, Singapore, 2011.

[40] H. Liebl, Applied Charged Particle Optics, vol. 2012, Springer, 2008.

[41] T. Zouros, "Theoretical investigation of the energy resolution of an ideal hemispherical deflector analyzer and its dependence on the distance from the focal plane," Journal of Electron Spectroscopy and Related Phenomena, vol. 152, no. 1, pp. 67-77, 2006, Erratum in: vol. 153, pp. 102-107, 2006.

[42] D. P. Geng, Y. Yang, S. Liu, C. Hong, and X. Gao, "Design of an efficient monochromatic electron source for inverse photoemission spectroscopy," Chinese Physics C, vol. 38, no. 11, Article ID 118202, 2014.

[43] C. C. Farnell, C. C. Farnell, S. C. Farnell, and J. D. Williams, "Electrostatic analyzers with application to electric propulsion testing," in Proceedings of the 33rd International Electric Propulsion Conference (IEPC '13), IEPC-2013-300, George Washington University, Washington, DC, USA, October 2013.

[44] M. A. Mahjoub, G. Monier, C. Robert-Goumet, L. Bideux, and B. Gruzza, "New method for the determination of the correction function of a hemisperical electron analyser based on elastic electron images," Journal of Electron Spectroscopy and Related Phenomena, vol. 197, pp. 80-87, 2014.

[45] C. Lupulescu, T. Arion, U. Hergenhahn et al., "IDEEAA: a novel, versatile apparatus for electron spectroscopy," Journal of Electron Spectroscopy and Related Phenomena, vol. 191, pp. 104-111, 2013.

[46] D. A. Dahl, "SIMION for the personal computer in reflection," International Journal of Mass Spectrometry, vol. 200, no. 1-3, pp. 3-25, 2000. 
[47] M. O. A. El Ghazaly, S. M. Alshammari, C. P. Welsch, and H. H. Alharbi, "Design of a novel electrostatic ion storage ring at KACST," Nuclear Instruments and Methods in Physics Research, Section A: Accelerators, Spectrometers, Detectors and Associated Equipment, vol. 709, pp. 76-84, 2013.

[48] M. I. Yavor, W. R. Plaß, T. Dickel, H. Geissel, and C. Scheidenberger, "Ion-optical design of a high-performance multiplereflection time-of-flight mass spectrometer and isobar separator," International Journal of Mass Spectrometry, 2015.

[49] P. Zhang, C. Xu, Y. Tang, and X. Chen, "Point-to-point focusing equation for double toroidal analyzer," Journal of Electron Spectroscopy and Related Phenomena, vol. 193, pp. 39-42, 2014.

[50] T. J. M. Zouros, O. Sise, F. M. Spiegelhalder, and D. J. Manura, "Investigation of the accuracy of ion optics simulations using Kepler orbits in a spherical capacitor," International Journal of Mass Spectrometry, vol. 261, no. 2-3, pp. 115-133, 2007.

[51] T. J. M. Zouros and E. P. Benis, "Optimal energy resolution of a hemispherical analyzer with virtual entry," Applied Physics Letters, vol. 86, no. 9, Article ID 094105, 2005.

[52] J. A. Simpson, "High resolution, low energy electron spectrometer," Review of Scientific Instruments, vol. 35, no. 12, pp. 16981704, 1964.

[53] K. Busch, "Space charge in mass spectrometry," Spectroscopy, vol. 19, no. 6, pp. 35-38, 2004.

[54] K. P. Grzelakowski and R. M. Tromp, “Temporal and lateral electron pulse compression by a compact spherical electrostatic capacitor," Ultramicroscopy, vol. 130, pp. 36-43, 2013. 

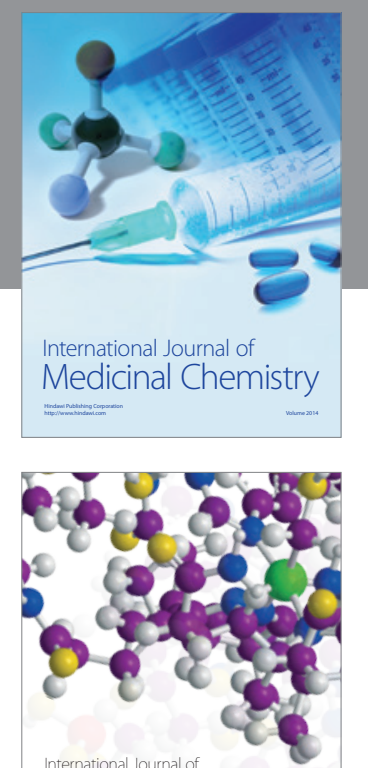

\section{Carbohydrate} Chemistry

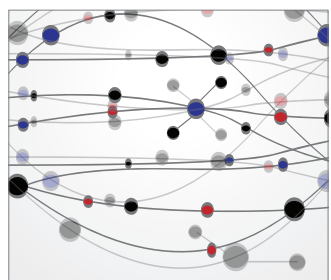

The Scientific World Journal
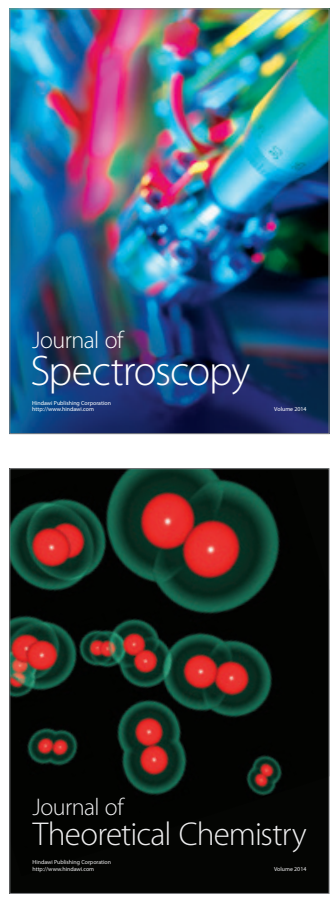
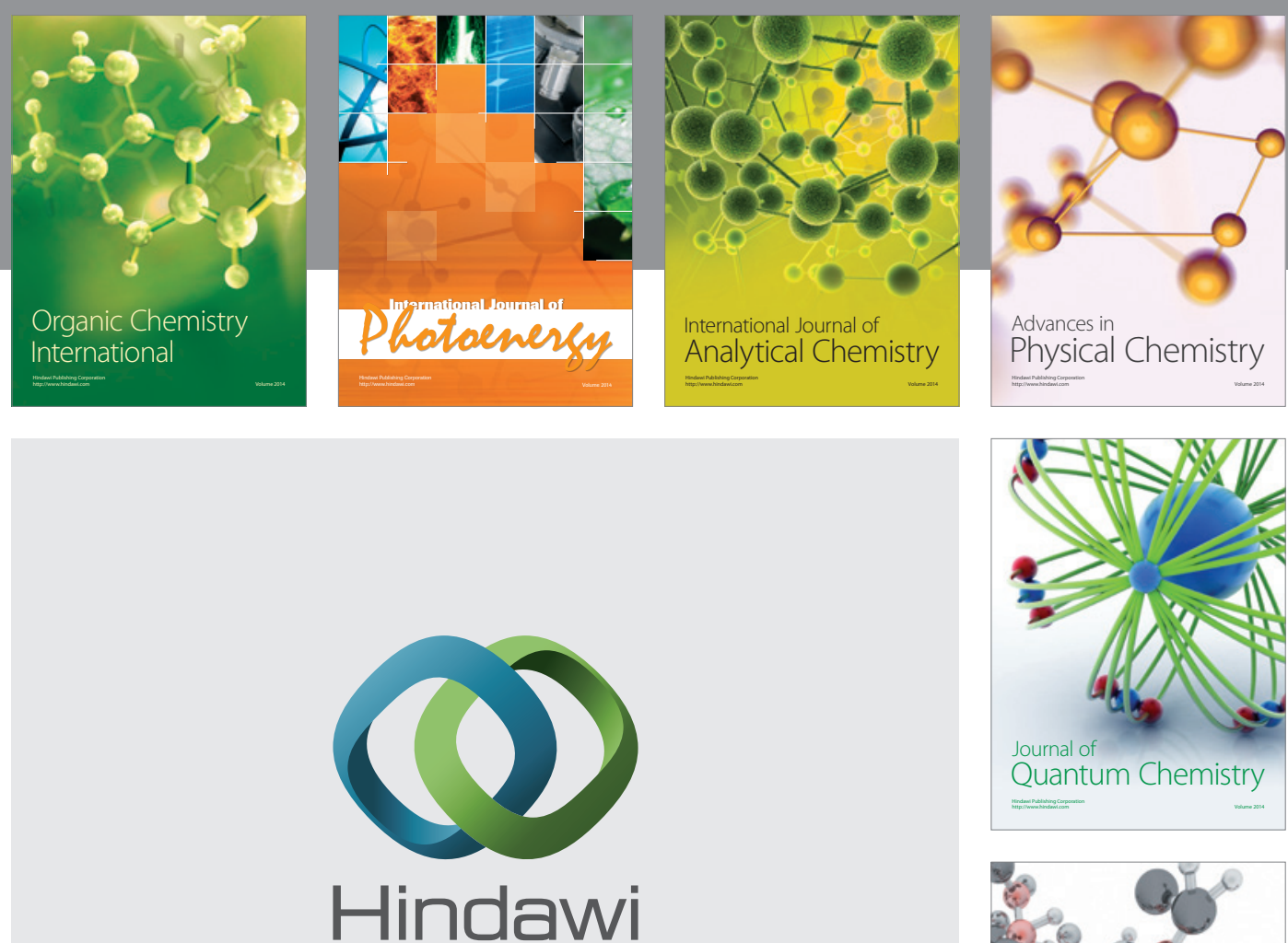

Submit your manuscripts at

http://www.hindawi.com

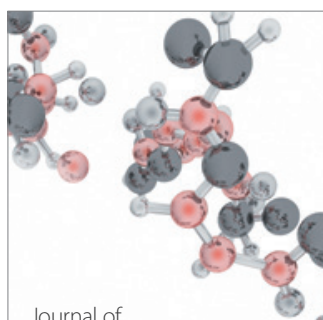

Analytical Methods

in Chemistry

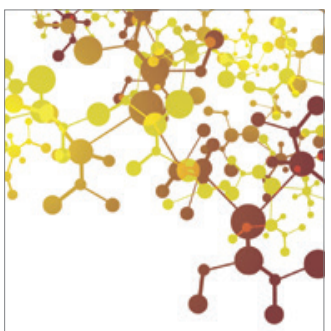

Journal of

Applied Chemistry

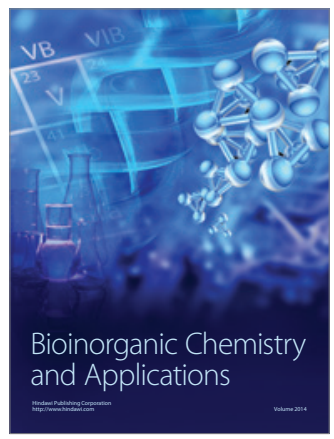

Inorganic Chemistry
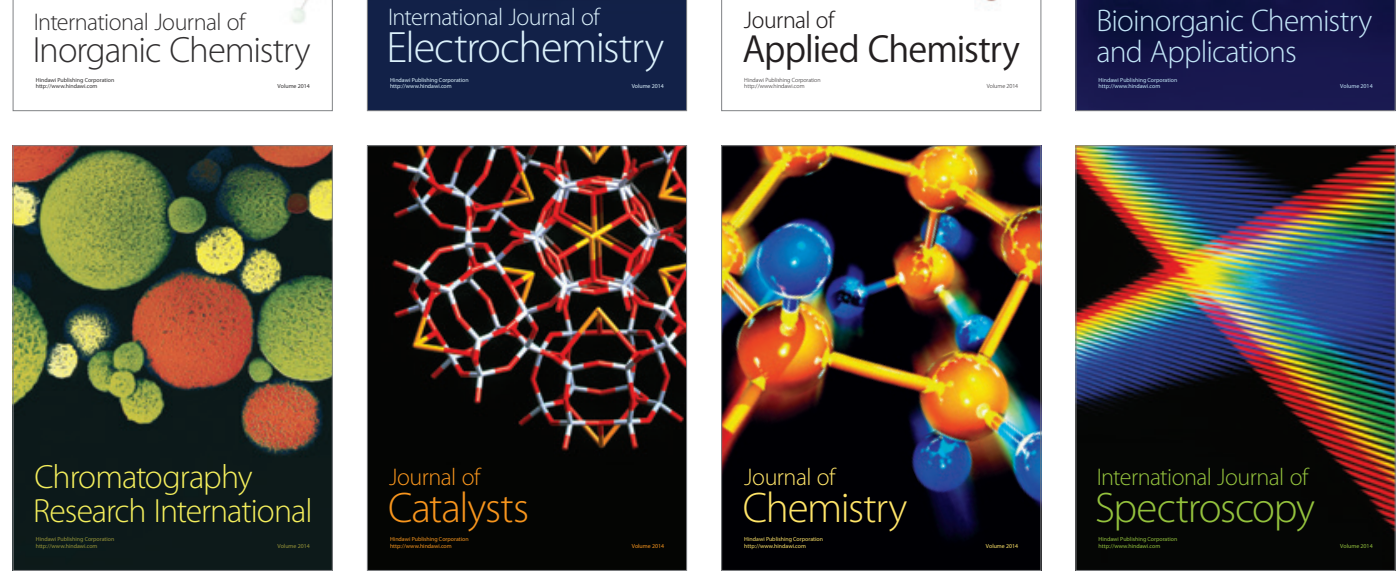